\title{
REVIEW ARTICLE OPEN Transfer printing techniques for flexible and stretchable inorganic electronics
}

\author{
Changhong Linghu' ${ }^{1}$, Shun Zhang ${ }^{1}$, Chengjun Wang ${ }^{1}$ and Jizhou Song ${ }^{1}$
}

Transfer printing is an emerging deterministic assembly technique for micro-fabrication and nano-fabrication, which enables the heterogeneous integration of classes of materials into desired functional layouts. It creates engineering opportunities in the area of flexible and stretchable inorganic electronics with equal performance to conventional wafer-based devices but the ability to be deformed like a rubber, where prefabricated inorganic semiconductor materials or devices on the donor wafer are required to be transfer-printed onto unconventional flexible substrates. This paper provides a brief review of recent advances on transfer printing techniques for flexible and stretchable inorganic electronics. The basic concept for each transfer printing technique is overviewed. The performances of these transfer printing techniques are summarized and compared followed by the discussions of perspectives and challenges for future developments and applications.

npj Flexible Electronics (2018)2:26; doi:10.1038/s41528-018-0037-x

\section{INTRODUCTION}

The last decade has witnessed the fast progresses and great achievements of flexible and stretchable inorganic electronics, which removes the planar, rigid, and brittle design constraints associated with conventional electronics via the integration of hard inorganic semiconductor materials in delicate structural layouts with flexible substrates. ${ }^{1-5}$ This technology has enabled many novel applications that are impossible for conventional electronics, such as curvilinear electronics, ${ }^{6-8}$ bio-integrated electronics, ${ }^{9-11}$ epidermal electronics, ${ }^{12-14}$ transient electronics, ${ }^{15-18}$ deformable opto-electronics, ${ }^{19-24}$ and many others. ${ }^{25-}$

27 Figure 1 shows some examples of flexible and stretchable inorganic devices with performance equal to those fabricated by established conventional technologies using well-developed inorganic semiconductor and metal materials, but in foldable, stretchable and curvilinear format. These examples include the stretchable and foldable Si-CMOS circuit (Fig. 1a), ${ }^{28}$ curvilinear electronics (a hemispherical electronic eye camera in Fig. $1 b^{6}$ ), biointegrated electronics (a multifunctional medical balloon catheter in Fig. $1 c^{9}$ and an ultrathin conformal bio-integrated neural electrode array in Fig. $1 \mathrm{~d}^{29}$ ), epidermal electronics (Fig. 1e), ${ }^{14}$ transient electronics (Fig. 1f) ${ }^{16}$ and deformable opto-electronics (a bendable photovoltaics module in Fig. $1 \mathrm{~g}^{23}$ an highly stretchable AllnGaP $\mu$-ILEDs array in Fig. $1 \mathrm{~h}^{30}$ and a mechanically flexed array of ultrathin, microscale, blue LEDs in Fig. $1 \mathrm{i}^{19}$ ).

All examples in Fig. 1 require the integration of inorganic semiconductor or metal materials with flexible polymeric substrates. However, the inorganic semiconductor or metal materials cannot be directly fabricated on flexible polymeric substrates using conventional fabrication technologies because the flexible polymeric substrates are not able to withstand the extreme processing conditions such as high temperature or chemical etching. A kind of manufacturing process of a flexible electronics system begins with independent fabrication of devices on wafer/ donor substrate followed by assembling them onto a flexible/ stretchable substrate together. Transfer printing technique, enabling the solid objects to be transferred from a donor substrate to a receiver substrate in a high yield manner, provides the most promising solution to this assembly process. ${ }^{31}$ This approach separates the fabrication substrates with the application substrates, bypassing the incompatibility problem of the polymeric substrates with conventional fabrication technologies which have a mature, established commercial infrastructure, thereby accelerating the commercialization of flexible and stretchable inorganic electronics.

The examples in Fig. 1 have demonstrated the extraordinary capabilities of transfer printing technique to deterministically assemble a myriad of materials (also called inks) into spatially organized, functional arrangements onto various substrates for flexible and stretchable inorganic electronics. The inks for flexible and stretchable inorganic electronics in transfer printing include hard inorganic materials (e.g., inorganic semiconductors ${ }^{17,32}$ and metals ${ }^{33,34}$ ), integrated inorganic devices (e.g., inorganic thin film transistors-TFTs, ${ }^{32}$ inorganic light emitting diodes-ILEDs ${ }^{20}$, and solar cells ${ }^{23,24}$ ) and fully integrated inorganic circuits. ${ }^{2,28,35}$ It should be noted that the inks for transfer printing are not limited to inorganic materials, but can also be other materials such as carbon materials (e.g., graphene ${ }^{31,36}$ ), organic materials (e.g., organic semiconductors ${ }^{37,38}$ ) and cell-sheet-integrated devices for flexible bioelectronics. ${ }^{39,40}$ There exist several reviews of transfer printing techniques focusing on materials and applications. ${ }^{31,41-43}$ This paper reviews the advances of transfer printing techniques for flexible and stretchable inorganic electronics (i.e., transfer printing techniques for inorganic semiconductor and metal materials, devices and circuits) from a methodological view. Fundamentals of transfer printing techniques are first introduced followed by the overview of various transfer printing techniques. These transfer printing techniques are classified based on their

\footnotetext{
${ }^{1}$ Department of Engineering Mechanics, Soft Matter Research Center, and Key Laboratory of Soft Machines and Smart Devices of Zhejiang Province, Zhejiang University, 310027 Hangzhou, China

Correspondence: Jizhou Song (jzsong@zju.edu.cn)
}

Received: 15 April 2018 Revised: 21 July 2018 Accepted: 31 July 2018

Published online: 08 October 2018 
(a)

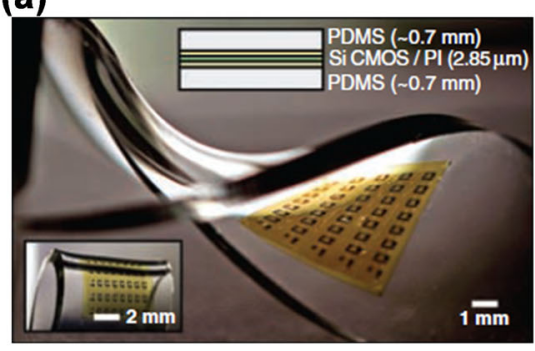

(d)

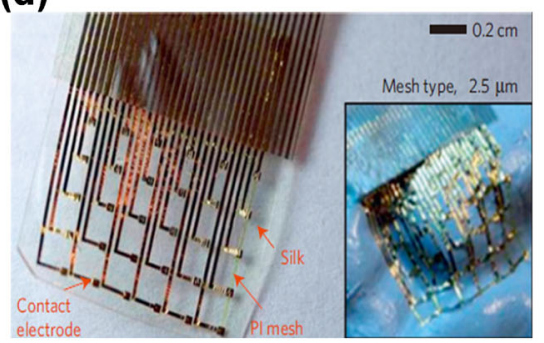

(g)

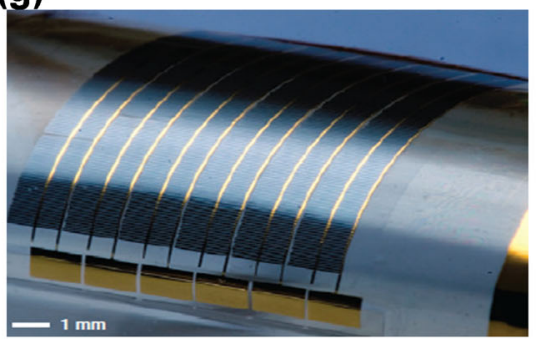

(b)

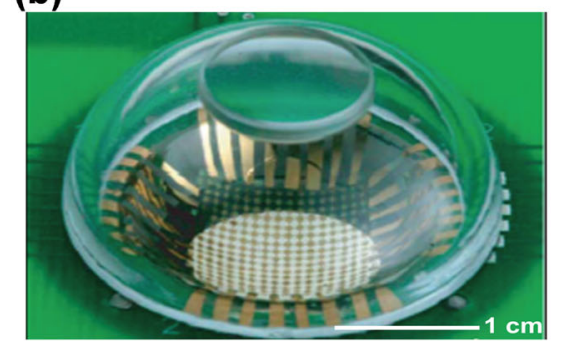

(e)

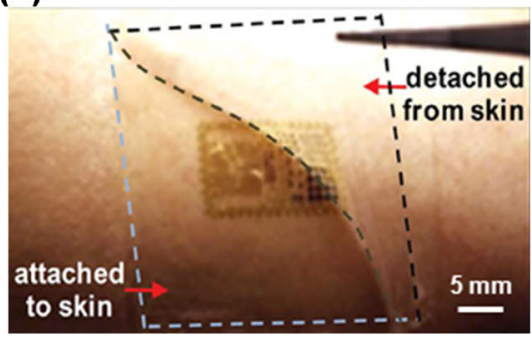

(h)

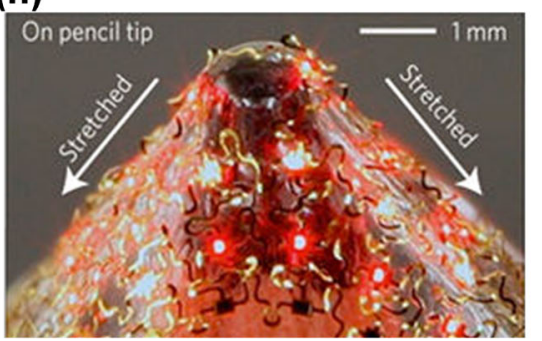

(c)

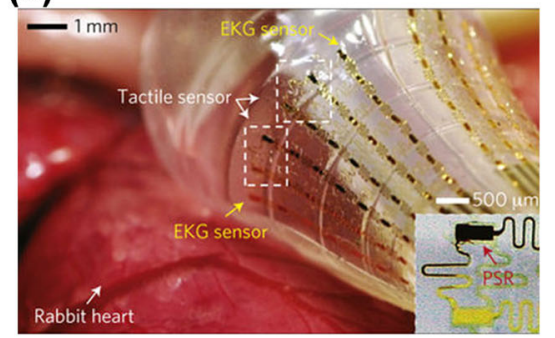

(f)

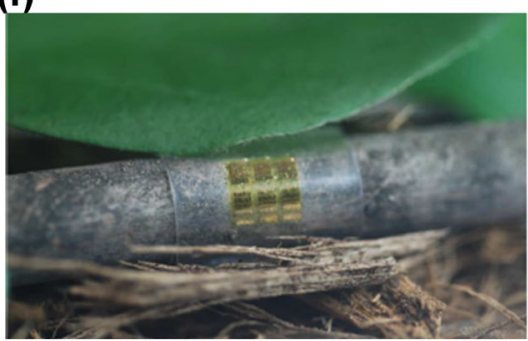

(i)

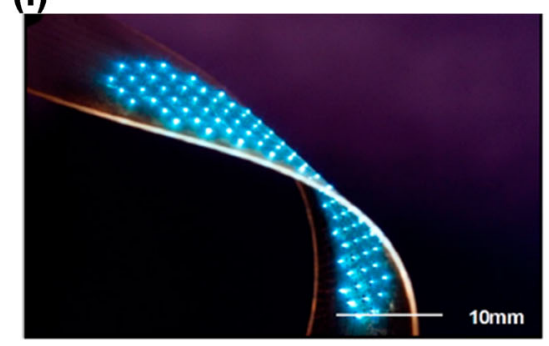

Fig. 1 Flexible and stretchable inorganic electronic devices enabled by transfer printing techniques. a Stretchable and foldable Si-CMOS circuit transferred onto a poly(dimethylsiloxane) (PDMS) substrate. Reprinted with permissions from ref. ${ }^{28}$. b A hemispherical electronic eye camera based on compressible silicon optoelectronics. The silicon optoelectronics are transferred from a flat donor substrate and printed onto a hemispherical glass lens substrate using a hemispherical PDMS transfer element. Reprinted with permissions from ref. ${ }^{6}$. c Multifunctional balloon catheters with biocompatible interfaces integrated directly by transfer printing for cardiac electrophysiological mapping and ablation therapy in its inflated state. Reprinted with permissions from ref. ${ }^{9}$. d Ultrathin conformal bio-integrated neural electrode arrays transferprinted onto a dissolvable silk substrate. Reprinted with permissions from ref. ${ }^{29}$. e Multifunctional epidermal electronic systems partially peeled away from the skin. Reprinted with permissions from ref. ${ }^{14}$. $\mathbf{f}$ A photograph of an array of GalnP/GaAs heterojunction bipolar transistors transfer-printed onto a biodegradable cellulose nanofibril substrate and wrapped around a tree stick with a $\sim 3 \mathrm{~mm}$ radius. Reprinted with permissions from ref. ${ }^{16} \mathbf{g}$ A bendable photovoltaics module that use large-scale arrays of silicon solar microcells created from bulk wafers and integrated in sparse spatial layouts on foreign substrates by transfer printing. Reprinted with permissions from ref. ${ }^{23} \mathbf{h}$ Optical images of an array of AllnGaP $\mu$-ILEDs $(6 \times 6)$ transferred onto a PDMS substrate and tightly stretched on the sharp tip of a pencil. Reprinted with permissions from ref. ${ }^{30} \mathbf{i}$ Image of a mechanically flexed array of ultrathin, microscale, blue LEDs printed from a source wafer onto a thin strip of plastic. Reprinted with permissions from ref. ${ }^{19}$

basic concepts and working principles, and then overviewed briefly. Finally, the performances of transfer printing techniques are compared, and some perspectives and challenges are discussed for future developments and applications.

\section{Fundamentals of transfer printing}

The most general form of the transfer printing process utilizes a soft, elastomeric stamp to mediate physical mass transfer of microdevices (usually termed as inks) between a donor substrate and a secondary, receiver substrate, as shown in Fig. $2{ }^{4}{ }^{44}$ It typically includes two steps: retrieval/pick-up of inks from the donor substrate and printing/delivery of inks onto the receiver substrate. At the beginning of the retrieval process, the stamp is brought into contact with the donor substrate, on which the inks are prepared in ordered and releasable manners usually through wet chemical etching ${ }^{41,45}$ or dry etching (e.g., laser lift-off ${ }^{46,47}$ ). For example, the wet chemical etching generates releasable inks via the etching of a sacrifice layer between the inks and substrate but with appropriately defined anchor structures to retain the lithographically defined spatial layouts of elements. A proper preload is applied on the stamp to ensure the conformal contact between the stamp and inks, which provides enough adhesion to retrieve inks from the donor substrate. The retrieval process can be either non-selective in a massively parallel way ${ }^{48,49}$ for high throughput or selective for precise manipulation on an individual ink or several inks. ${ }^{20,21,50}$ The inked stamp is then brought into contact with the receiver substrate followed by the modulation of the stamp/ink adhesion to print inks onto the receiver substrate. The removal of the stamp completes the transfer printing process. The printing mode can also be non-selective ${ }^{48}$ or selective. ${ }^{51,52}$

The underlying physics associated with the transfer printing process is within the scope of fracture mechanics, which involves a system of three layers (stamp/ink/substrate) with two interfaces (stamp/ink and ink/substrate interfaces) as shown in Fig. 2b. ${ }^{44}$ The competing fracture between the stamp/ink interface and the ink/ substrate interface determines whether retrieval or printing occurs. During the retrieval process, the stamp/ink interface should be stronger than the ink/substrate interface such that the inks can be retrieved by the stamp. During the printing process, 
(a)

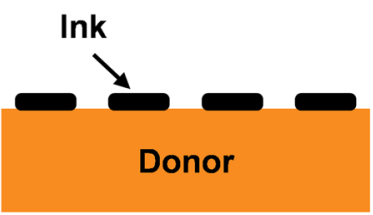

1) Inks on donor

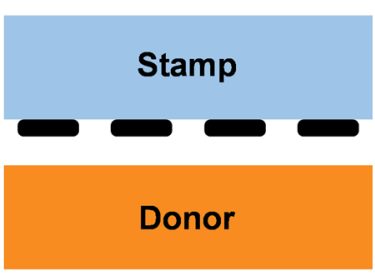

2) Retrieval

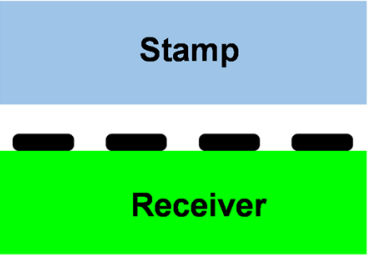

3) Printing (b)

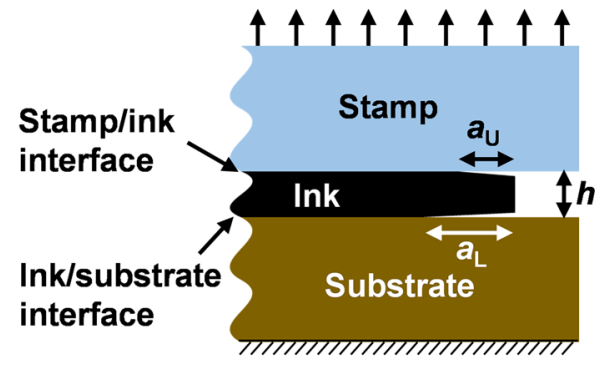

(c)

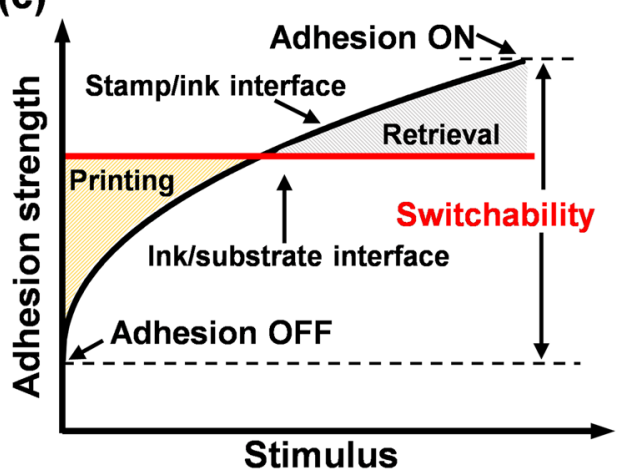

Fig. 2 Operating process and principles of transfer printing. a Illustration of the transfer printing process. (1) Inks are prepared on the donor substrate in a releasable manner. (2) Retrieval process: an elastomer stamp is used to retrieve the inks. (3) Printing process: inks are printed onto the receiver substrate. Adapted with permission from ref. ${ }^{44} \mathbf{b}$ The two interfaces in the stamp/ink/substrate structure. Adapted with permission from ref. ${ }^{44}$ c Adhesion strength modulated by external stimulus, showing the high (ON) and low (OFF) adhesion state and the switchability

the stamp/ink interface should be weaker than the ink/substrate interface such that the inks can be released from the stamp. The yield of transfer printing depends critically on the ability to switch the adhesion between strong states and weak states for retrieval and printing, respectively. Usually, the adhesion strength at the ink/substrate interface is independent of external stimuli and is considered as a constant. Thus, the key to successful transfer printing becomes the adhesion modulation of the stamp/ink interface. Figure $2 \mathrm{c}$ illustrates the basic principle for the transfer printing technique: retrieval under the strong stamp/ink adhesion state and printing under the weak stamp/ink adhesion state, where the adhesion strength at the ink/substrate interface (red solid line) remains a constant while the adhesion strength at the stamp/ink interface (black solid line) is modulated by the external stimuli such as peel velocity ${ }^{48,53,54}$ and lateral movement. ${ }^{55}$ The adhesion switchability, which is defined as the ratio of the maximum adhesion strength to the minimum adhesion strength, can be used to evaluate the adhesion modulation performance.

Based on the principles of the adhesion modulation of the stamp/ink interface, the transfer printing techniques are classified into surface chemistry and glue assisted transfer printing technique, kinetically controlled transfer printing technique, laser-driven non-contact transfer printing technique, geckoinspired transfer printing technique (representing a set of techniques with fibrillar surface assisted with specific manipulation such as retraction angle or lateral movement), and aphidinspired transfer printing technique (representing a set of techniques based on the change of contact area).

Surface chemistry and glue assisted transfer printing technique To enhance the reliability of the retrieval and printing processes, surface chemical modification or glue is used to modulate the interfacial adhesion strength. Figure $3 a^{56}$ shows a typical surface chemistry and glue assisted transfer printing process with the retrieval assisted with surface chemistry and printing assisted with glue. The strong stamp/ink adhesion required for retrieval is realized through the $\mathrm{Si}-\mathrm{O}-\mathrm{Si}$ chemical bonding between the surface of the slightly oxidized PDMS stamp ${ }^{45,56}$ and the fresh $\mathrm{SiO}_{2}$ film coated on the target inks via a condensation reaction. ${ }^{57}$ The printing is realized through the increase of the ink/substrate interfacial adhesion by coating a thin layer of glue, which is usually in liquid/uncured ${ }^{56,58,59}$ state, partially cured state, ${ }^{58,60}$ or cured state with low modulus. ${ }^{32,61}$ The uncured or partial cured glue is further cured by heating ${ }^{59,62}$ or UV light exposing ${ }^{45,56,62}$ to enhance the bonding between the inks and the receiver substrate. Although the surface chemistry and glue can enhance the reliability of retrieval ${ }^{45,56}$ and printing, ${ }^{58,63}$ the density of chemical bonds must be carefully designed to allow the successful removal of the inks from the stamp. Moreover, the need of additional $\mathrm{SiO}_{2}$ layer to enhance stamp/ink adhesion complicates the process and may cause the frequent replacement of stamps due to the chemical reaction of $\mathrm{SiO}_{2}$ layer after transfer printing and the contamination of glues to the stamp.

Surface treatment of the donor substrate is also a critical factor for transfer printing process. Some materials have strong adhesion with the donor substrate, so that appropriate surface treatment, such as self-assembled monolayers, is required to weaken the adhesion between the ink and the donor substrate. For example, in the case of transfer printing of colloidal quantum dots, ${ }^{21,64}$ the interaction between the nanoparticles and the substrates should be within the proper range for efficient transfer printing and specific surface treatment (e.g., coating of octadecyltrimethosysilane self-assembled monolayers ${ }^{21,64}$ ) is often required before coating solution of nanoparticles on the donor substrate.

Another type of transfer printing technique involving surface chemistry with a much more simplified process is the tape transfer printing, where solvent releasable tapes ${ }^{13,65,66}$ or thermal releasable tapes ${ }^{10,67}$ are used as stamps. Figures $3 \mathrm{~b}-1^{66}$ shows the typical transfer printing process based on a commercially available solvent releasable adhesive tape to retrieve and print devices with high yields. The high adhesion strength between the tape and inks ensures the retrieval of inks from the donor substrate with a 
(a)

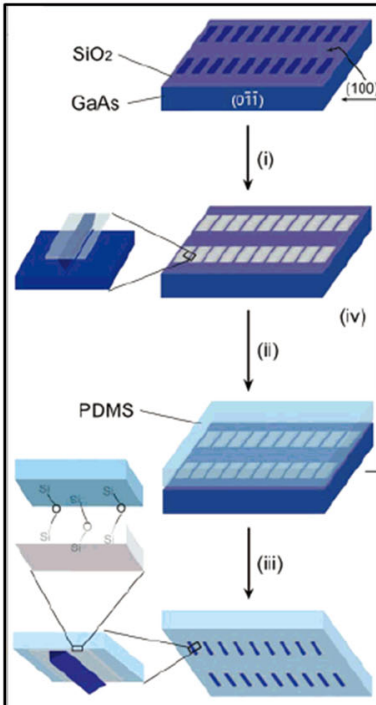

1) Retrieval assisted with surface chemistry (b)

1)

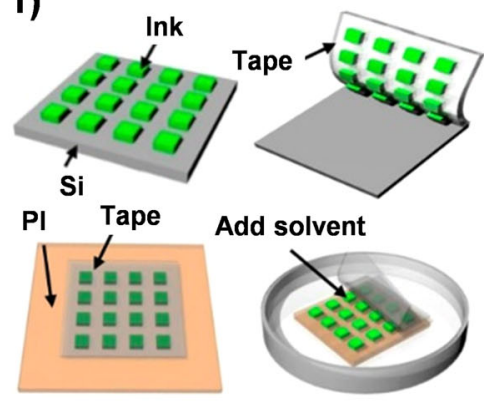

2)

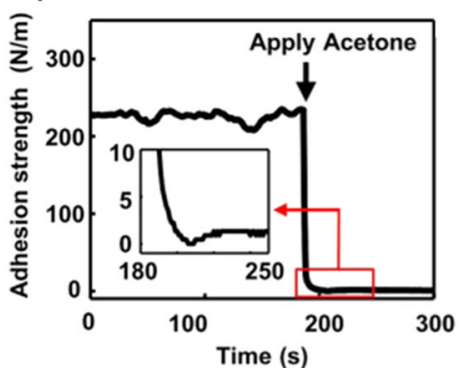

(c)

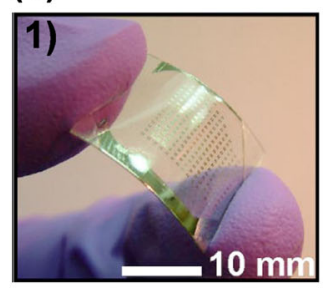

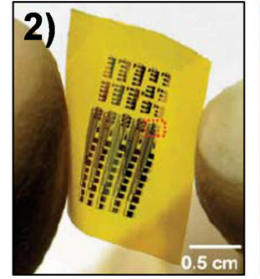

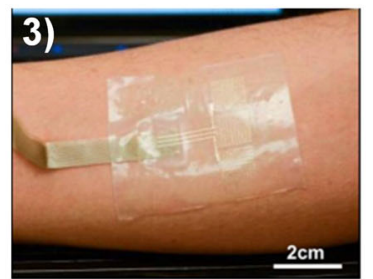

Fig. 3 Surface chemistry and glue assisted transfer printing techniques. a Schematic illustration of the transfer printing process of GaAs wire arrays onto plastic substrates. (1) Retrieval process utilizing the Si-O-Si chemical bonding between the surface of the slightly oxidized PDMS stamp and the fresh $\mathrm{SiO}_{2}$ film coated on the GaAs wire arrays. (2) The steps involved in the transfer process of GaAs wire arrays from PDMS stamp to PET sheet coated with a thin layer of PU. Reprinted with permissions from ref. ${ }^{56} \mathbf{b}$ Illustration of the transfer printing process of the solvent releasable tape and plot of measured adhesive strength of the $3 \mathrm{M} 3850$ tape before and after introducing acetone under $180^{\circ}$ peeling test. Adapted with permission from ref. ${ }^{66}$ c Devices and structures enabled by surface chemistry and glue assisted transfer printing techniques. (1) A high performance thin film transistor built on a photosensitive epoxy coated PET substrate. Reprinted with permissions from ref. ${ }^{60}$ (2) Array of 3D silicon n-channel metal oxide semiconductor inverters on a polyamic acid coated PI substrate. Reprinted with permissions from ref. ${ }^{68}$ (3) An EMG sensor mounted on the skin of forearm for measurement enabled by solvent releasable tape. Reprinted with permissions from ref. ${ }^{66}$

high reliability. During the printing process, the introducing of solvent significantly reduces the adhesion strength at the stamp/ ink interface to almost zero by decomposing the interface or even the whole tape, which ensures the printing of inks onto the receiver substrate with a high reliability. Figures $3 b-2^{66}$ shows the adhesive strength of the $3 \mathrm{M} 3850$ tape on glass before and after introducing acetone, which clearly indicates the modulation effectiveness of the adhesion strength via surface chemistry. Although the tape transfer printing provides a simple but highly reliable approach for the heterogeneous integration of materials, it typically leaves certain residuals on inks, which may degrade the device performance.

Figure $3 c$ shows some devices enabled by the surface chemistry and glue assisted transfer printing techniques: (1) a high performance thin film transistor built on a photosensitive epoxy coated PET substrate, $^{60}(2)$ an array of 3D silicon n-channel metal oxide semiconductor inverters on a polyamic acid coated PI substrate, ${ }^{68}$ and (3) an EMG sensor ${ }^{66}$ mounted on the skin of forearm. Although the surface chemistry and glue assisted transfer printing techniques are simple and straightforward, they have their intrinsic limitations such as surface contamination which may cause the frequent replacement of stamps and may degrade the device performance. To overcome these limitations, advanced transfer printing techniques based on tunable and reversible dry adhesion have been developed and will be discussed in the following sections.

Kinetically controlled transfer printing technique

A powerful and versatile transfer printing technique, kinetically controlled transfer printing technique, ${ }^{48,53,54}$ takes advantage of the rate-dependent adhesion effect of viscoelastic stamps to retrieve the inks from the donor substrate at a high velocity $(\sim 10 \mathrm{~mm} / \mathrm{s}$ or greater) and print the inks onto the receiver substrate at low velocity $(<1 \mathrm{~mm} / \mathrm{s})$, as shown in Fig. 4a. ${ }^{48}$ Figure $4 b^{48}$ shows the rate dependency of the critical energy release rate for steel/PDMS interface. Due to the viscoelastic effect of PDMS, the critical energy release rate increases monotonically with the separation speed, which enables the adhesion modulation at the stamp/ink interface by controlling the peeling velocity.

Feng et al. ${ }^{53}$ studied the kinetically controlled transfer printing process and modeled the peeling process of the stamp from the substrate as the steady propagation of interfacial cracks to establish the criterion for predicting whether retrieval or printing occurs as shown in Fig. 4c. The critical energy release rate $G_{\text {crit }}^{\text {inksubstrate }}$ of the ink/substrate interface is rate-independent since both the ink and the substrate are elastic, while the critical energy release rate $G_{\text {crit }}^{\text {ink/stamp }}(v)$ of the stamp/ink interface is rate dependent due to the viscosity of the stamp. The condition $G_{\text {crit }}^{\text {ink substrate }}=G_{\text {crit }}^{\text {ink/stamp }}(v)$, which corresponds to the intersection of the two curves in Fig. $4 c$, gives the critical peeling velocity $v_{c}$ separating the retrieval and printing regimes. The retrieval occurs when the peeling velocity is larger than $v_{c}$, while the printing occurs when the peeling velocity is smaller than $v_{c}$. Due to the limited range of modulation in adhesion strength, the kinetically controlled transfer printing may fail to retrieve the inks from the donor substrate for strong ink/substrate interface and fail to print the inks onto the receiver substrate for weak ink/substrate interface. In order to improve the yields of printing for weak ink/substrate interface, Kim et al. ${ }^{54}$ introduced narrow, shallow reliefs on the stamp surface to reduce the contact area between the stamp and ink, thus to reduce the critical energy release rate of the stamp/ink interface. Feng et al. ${ }^{69}$ improved the adhesion modulation method by directional peeling this type of stamp: peeling the stamp perpendicular and parallel to the surface reliefs 
(a)

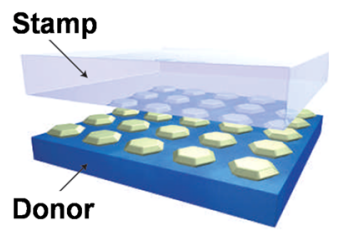

1) Prepare donor substrate; apply rubber stamp

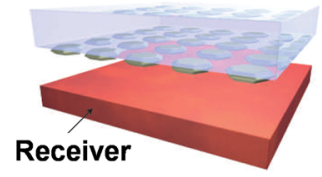

3) Apply inked stamp to receiving substrate

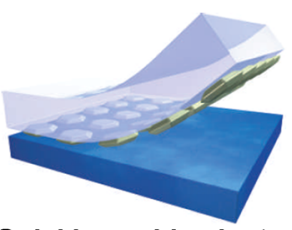

2) Quickly peel-back stamp; grab objects off the donor

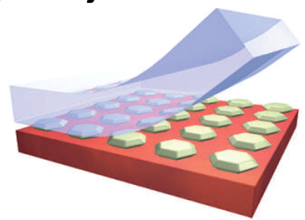

4) Slowly peel-back stamp; print objects onto receiver. (b)

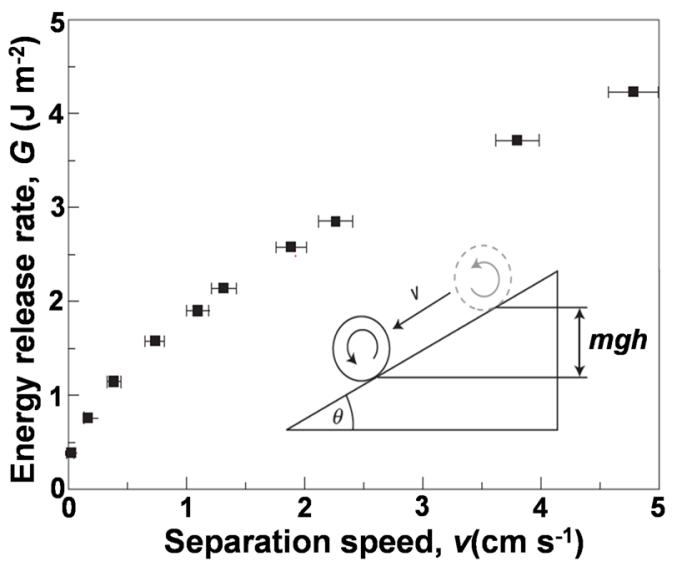

(d)
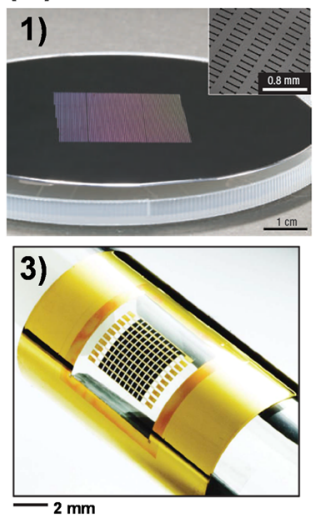
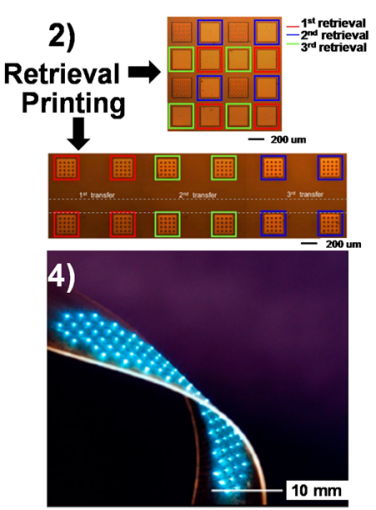

Peel velocity

Fig. 4 Kinetically controlled transfer printing technique. a Schematic illustration of the transfer printing process: retrieval at high velocity and printing at low velocity. Reprinted with permissions from ref. ${ }^{48} \mathbf{b}$ Rate dependency of the critical energy release rate obtained by rolling down a steel cylinder on an inclined PDMS slab. Reprinted with permissions from ref. ${ }^{48} \mathbf{c}$ The criterion for kinetically controlled transfer printing. Adapted with permission from ref. ${ }^{53}$ d Structures, electronics and optoelectronics enabled by kinetically controlled transfer printing technique. (1) Large scale $(30 \mathrm{~mm} \times 38 \mathrm{~mm})$ array of I-shaped silicon microstructures printed in ambient conditions directly onto a $100 \mathrm{~mm}$ $\mathrm{GaAs}$ wafer in a parallel way. Reprinted with permissions from ref. ${ }^{48}$ (2) Selective retrieval and non-selective printing in three sequences with area expansion of inorganic LEDs. Adapted with permission from ref. ${ }^{20}$ (3) Bendable GaAs solar cells arrays. Reprinted with permissions from ref. $^{70}$ (4) GaN LED arrays printed on a plastic substrate. Reprinted with permissions from ref. ${ }^{19}$

yields high and low critical energy release rate, respectively. Recently, the combination of the bending radius with the peeling velocity of a flat PDMS stamp has been shown to enhance the printing efficiency significantly. ${ }^{49}$

Kinetically controlled transfer printing technique has been widely used to fabricate flexible and stretchable inorganic electronics in a massively parallel way (Fig. $4 \mathrm{~d}-1)^{48}$ or selective mode (Fig. $4 d-2) .{ }^{20}$ Some devices enabled by kinetically controlled transfer printing technique are shown in Fig. $4 d-3$ for flexible GaAs solar cells arrays ${ }^{70}$ and Fig. $4 \mathrm{~d}-4$ for flexible GaAs LED arrays. ${ }^{19}$ Although the kinetically controlled transfer printing technique is simple and convenient, it has its limitations including (1) an additional instrument for velocity control is needed, (2) the adhesion strength at an extremely high peeling velocity is still relatively low, which is not desirable for a highly reliable retrieval, (3) the adhesion strength at an extremely low peeling velocity is still relatively high, which is not desirable for a highly reliable printing, and (4) the adhesion switchability is relative low (less than three ${ }^{71}$ for a flat stamp). A transfer printing technique eliminating the additional instrument with more controllable switching between strong and weak adhesion state is desired in practical applications.
Laser-driven non-contact transfer printing technique

A distinguished transfer printing technique achieving infinite adhesion switchability is the laser-driven non-contact transfer printing technique, ${ }^{72,73}$ which utilizes the large thermalmechanical mismatch at the stamp/ink interface upon heating up to over $275^{\circ} \mathrm{C}$ by a laser pulse to drive the delamination and allows non-contact printing with performance independent of the receiver substrate's geometry and properties. Figure $5 a^{72}$ illustrates a typical cycle for the laser-driven non-contact transfer printing process. The elastomeric PDMS stamp is aligned to and brought into contact with the donor substrate to retrieve the ink. Then the inked stamp is brought close to the receiver substrate (a few micrometers above the receiver) and a laser pulse is used to heat the stamp/ink interface. As the temperature increases, the energy release rate also increases as shown in Fig. $5 b .^{72}$ Once the energy release rate reaches the critical value, the ink starts to delaminate from the stamp and then drops onto the receiver substrate.

Li et al. ${ }^{7475}$ established a thermo-mechanical model to obtain the temperature increase in the system and the energy release rate at the crack tip. By equaling the energy release rate with the work of adhesion of the interface, a scaling law for the delamination time is obtained as $t_{\text {delamination }} / t_{0}=f\left(q_{\text {total }} / q_{0}, L_{\text {silicon }} / L_{0}\right)$, where 
(a)

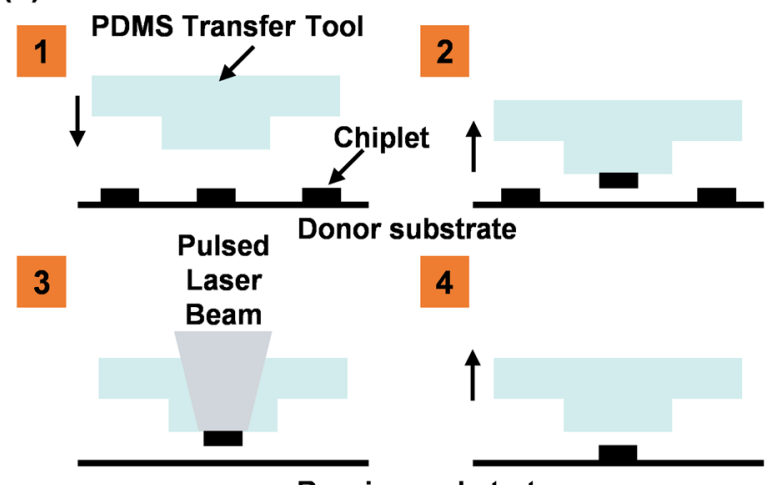

Receiver substrate (b)

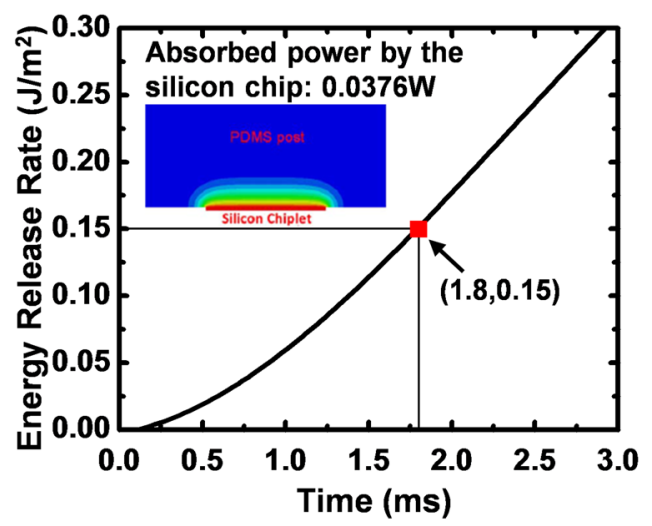

(c)

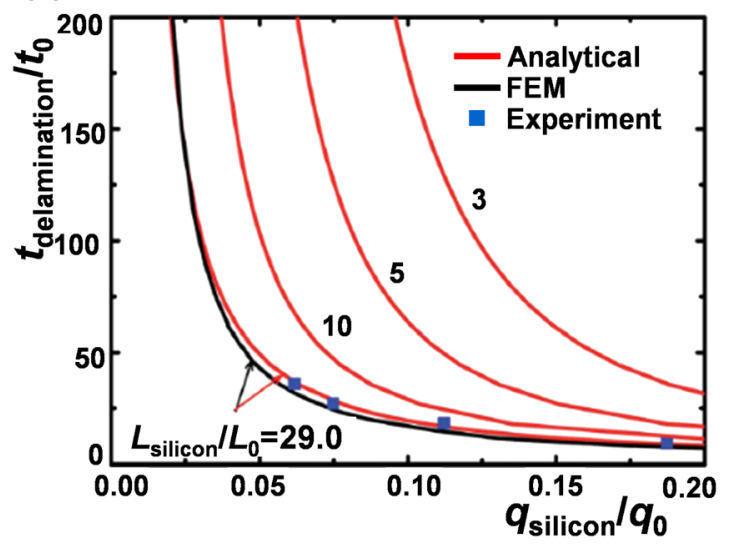

(d)
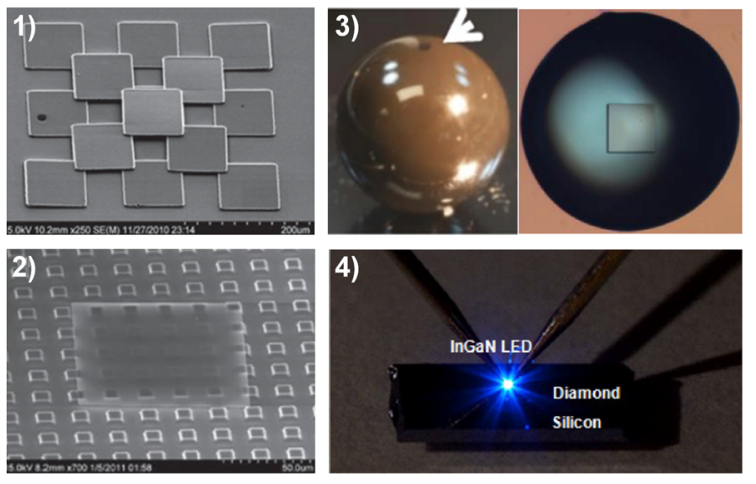

Fig. 5 Laser-driven non-contact transfer printing technique. a Illustration of the laser-driven non-contact transfer printing process. Adapted with permission from ref. ${ }^{72} \mathbf{b}$ Energy release rate at the crack tip versus time. Inset: temperature distribution in the stamp and the attached chiplet at $1.8 \mathrm{~ms}$. Adapted with permission from ref. ${ }^{72} \mathrm{c}$ The scaling law for the delamination of the stamp/silicon interface. Reprinted with permissions from ref. ${ }^{74} \mathbf{d}$ Structures fabricated using the laser-driven non-contact transfer printing technique. (1) Three-dimensional pyramid built with the same silicon squares. Reprinted with permissions from ref. ${ }^{72}$ (2) $100 \mu \mathrm{m} \times 100 \mu \mathrm{m} \times 0.32 \mu \mathrm{m}$ ultrathin Si square placed onto a structured substrate. Reprinted with permissions from ref. ${ }^{72}$ (3) Examples of printing on curved surfaces, (left) printing on a single $1 \mathrm{~mm}$ ceramic sphere and (right) printing onto a liquid NOA droplet. Reprinted with permissions from ref. ${ }^{73}$ (4) Functioning $\mu$-LED placed on a CVDgrown polycrystalline diamond on silicon substrate. Reprinted with permissions from ref. ${ }^{72}$

$t_{\text {delamination }}$ is the delamination time, $q_{\text {total }}$ is the total heat flux to the stamp/ink interface from the pulsed laser beam, $L_{\text {silicon }}$ is the width of the silicon chip (i.e., the ink), $t_{0}, q_{0}$, and $L_{0}$ represent the characteristic time, heat flux and length in the system, respectively. It is noticed that the delamination time depends only on two nondimensional parameters: the normalized total heat flux and the normalized silicon chip width. The analytical model agrees well with FEM and experiments as shown in Fig. $5 c^{74}$ The above analytical model accounts for the width effect of Si chip on the temperature distribution but not on the energy release rate, and therefore it is only valid for relative wide Si chips. This motivates the development of an accurate interfacial fracture mechanics model accounting for the width effect of the Si chip. ${ }^{76}$

During the printing process, the stamp does not contact directly with the receiver substrate, thus the success of the printing process is independent of the topography and properties of the receiving surface. Besides, a patterned stamp facilitates selective retrieval. The printing can be also selective due to the high spatial resolution of a laser. These superior capacities are demonstrated in Fig. $5 d$, where silicon squares are built into a three-dimensional pyramid (Fig. 5d-1), ${ }^{72}$ an ultra-thin silicon square $(0.32 \mu \mathrm{m})$ is transferred onto a structured substrate (Fig. $5 \mathrm{~d}-2){ }^{72}$ silicon squares are placed onto curvilinear surfaces such as a ceramic sphere and a liquid NOA droplet (Fig. 5d-3), ${ }^{73}$ and a functioning $\mu$ LED is placed on a CVD-grown polycrystalline diamond on silicon substrate (Fig. 5d-4). ${ }^{72}$ The laser-driven non-contact transfer printing technique is the only transfer printing technique that can be manipulated in a non-contact printing mode reported so far. The requirement of a high temperature increase (over $275^{\circ} \mathrm{C}$ ) at the stamp/ink interface for printing is detrimental and may cause surface damage to the stamp. ${ }^{52}$ To handle this problem, Eisenhaure and $\mathrm{Kim}^{51}$ designed a shape memory polymer (SMP) stamp with carbon black particles embedded within the stamp surface near to where it contacts the ink to absorb the incoming NIR laser. By imbedding laser-absorbing particles within the SMP matrix, the speed and localization of heat delivery are greatly enhanced. This method greatly reduces the required power input of laser and the risk of thermal damage.

\section{Gecko-inspired transfer printing technique}

Gecko's toe pad (Fig. 6a) ${ }^{77}$ is characterized by superior adhesion strength, reusability, tolerance to substrates, and easy detachment, ${ }^{77,78}$ which originate from the hierarchical, fibrillar structures $^{78,79}$ and the directional adhesion of the angled setae and spatulae $e^{78,80}$ controlled by mechanical deformations induced by vertical and lateral loading of its feet. Inspired by gecko's tunable and reversible adhesive pad, many gecko-inspired transfer printing techniques have been developed such as transfer printing technique using an angled microflap stamp, ${ }^{81}$ shear- 
(a)

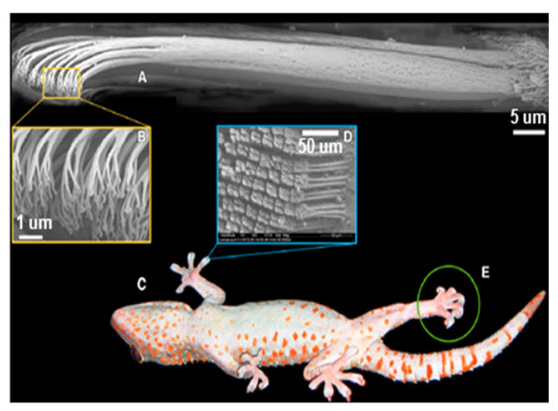

(b)
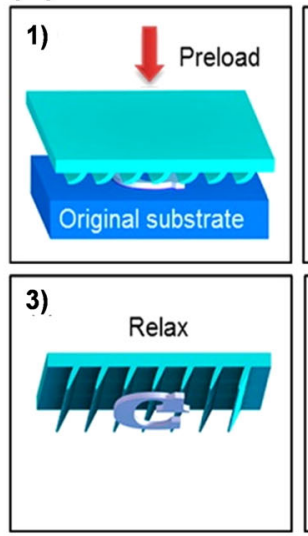

(c)

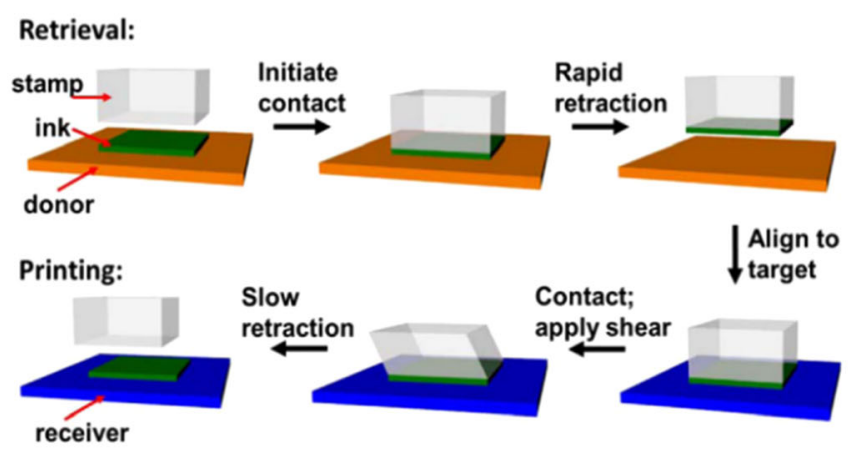

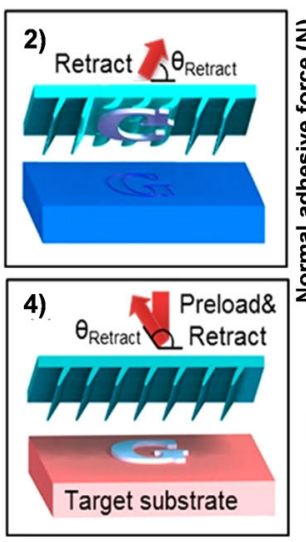
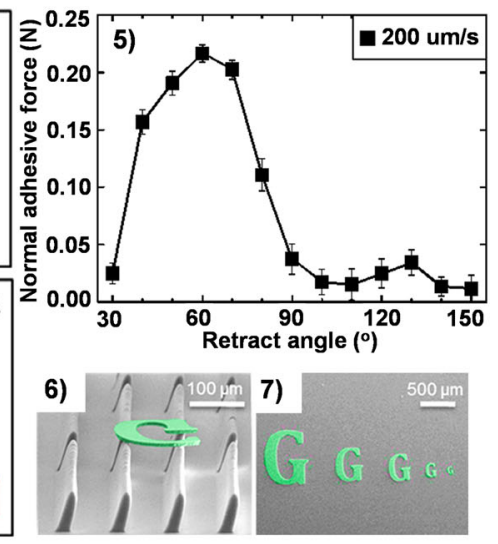

(d)

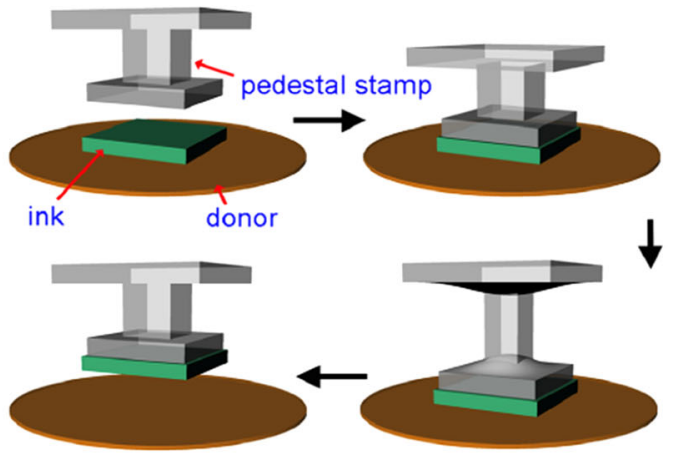

(e)
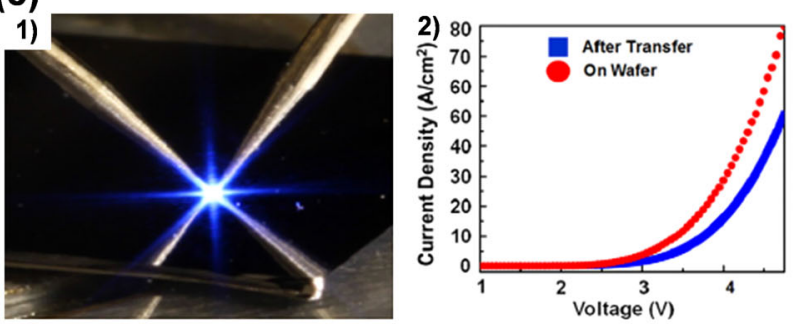

(f)
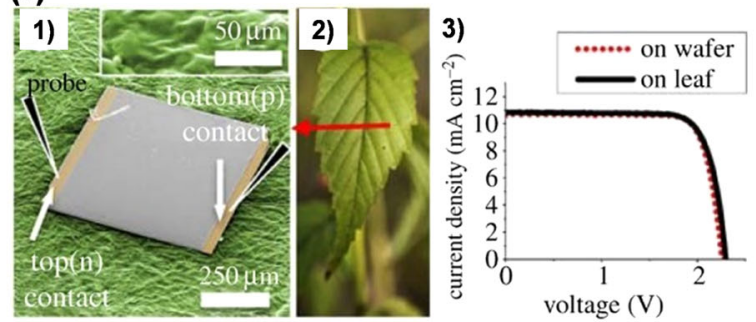

Fig. 6 Gecko inspired transfer printing techniques. a Gecko adhesive system. Adapted with permission from ref. ${ }^{77} \mathbf{b}$ Gecko-inspired transfer printing using an angled microflap stamp: (1-4) Transfer printing process; (5) Retraction-angle-dependency of the normal adhesive force and (6-7) capacity demonstrations of transfer printing. Adapted with permission from ref. ${ }^{81}$ c Shear-enhanced transfer printing technique with a lateral displacement to reduce the adhesion for printing. Reprinted with permissions from ref. ${ }^{55} \mathbf{d}$ Transfer printing technique using a pedestal stamp. Here the end plate on the post end enhances the adhesion, hence broadens the materials that can be transferred. Reprinted with permissions from ref. ${ }^{83}$ e (1) A $\mu$-ILED transfer-printed by a pedestal stamp and illuminated at the probe station and (2) the recorded I-V characteristics. The retrieval and printing with a pedestal stamp does not affect the performance of the $\mu$-ILED too much and the observed current densities are similar to those of the devices tested prior to printing. Reprinted with permissions from ref. ${ }^{83} \mathbf{f}(1)$ Colorized SEM image of a $2 \mathrm{~J}$ solar transfer-printed (2) on a leaf using the gecko seta array stamp. (3) The Current-voltage (I-V) characteristics of the solar microcells on the original substrate (GaAs wafer) and on the leaf. The electrical performances of the solar microcells on both surfaces are almost identical, showing that the transfer printing process using the gecko seta array stamp imposes little influence on the device. Reprinted with permissions from ref. 84

enhanced transfer printing technique, ${ }^{55}$ buckling-actuated transfer printing technique, ${ }^{82}$ transfer printing technique using a pedestal stamp, ${ }^{83}$ transfer printing technique using a gecko seta array stamp, ${ }^{84}$ etc.

Yoo et al. ${ }^{81}$ developed an angled microflap stamp for transfer printing with the process illustrated in Figs. 6b-1-4. This stamp with compliant microflaps on its surface possesses strong retraction angle dependent adhesion as shown in Fig. $6 b-5$. The adhesion strength reaches the maximum at a retraction angle of $\sim 60^{\circ}$ and the minimum at $\sim 100^{\circ}$, respectively, which indicates that the retraction angle can be utilized to control the adhesion switch between strong and weak state. Thus, the retrieval of inks from the donor substrate and printing of them onto the receiver substrate can be obtained at the retraction angle of $\sim 60^{\circ}$ and $\sim 100^{\circ}$, respectively. Due to the high compliance, the angled microflap stamp is able to transfer and print inks with complex geometries and various scales (Figs. 6b-6, 7).

The shear-enhanced transfer printing technique ${ }^{55}$ utilizes the stamp with vertical posts as illustrated in Fig. 6c. The retrieval occurs when the stamp is retracted rapidly and the printing is assisted with a lateral displacement. The applied lateral displacement generates a shear deformation in the stamp, which causes a 


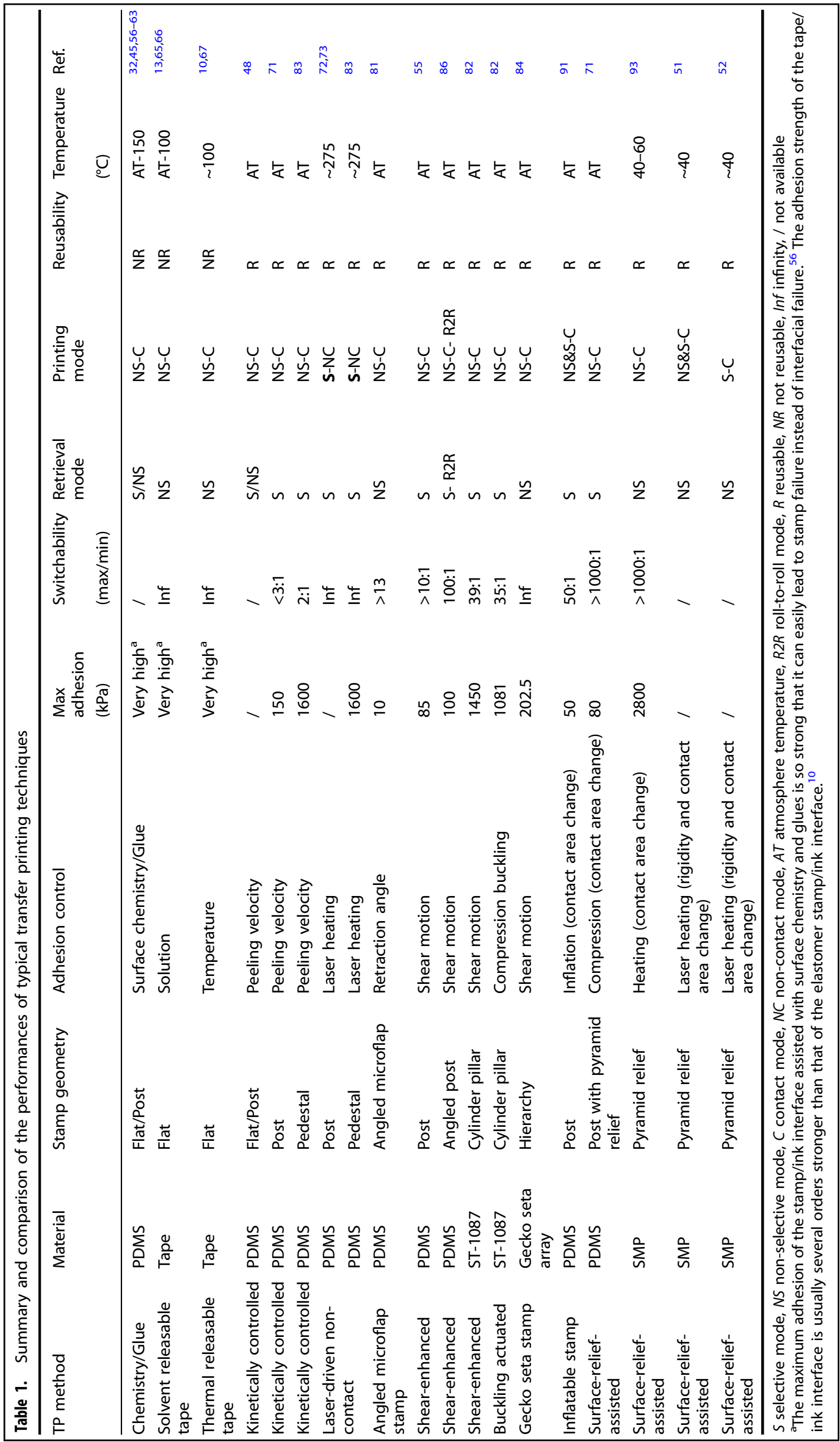


mixed mode loading at the interface. ${ }^{85}$ The resulting stress distribution at the stamp/ink interface becomes asymmetric and produces a larger normal stress at the edge, which leads to a larger strain energy release rate and enables easy delamination of the ink from the stamp. Yang et al. ${ }^{86}$ further designed a stamp with angled posts to improve the performance based on the stamp with vertical posts. The crack propagation is accelerated when the lateral movement is applied opposite to the inclination direction and frustrated along the other direction, leading to low and high adhesion strengths, respectively. This facilitates the transfer printing by simply choosing the retraction direction of the stamp. The retraction direction dependence of the angled post offers possibilities for high-throughput roll-to-roll (R2R) applications. The typical maximum adhesion strength and adhesion switchability for shear-enhanced transfer printing technique using PDMS with vertical posts are $100 \mathrm{kPa}$ and 100 , respectively. ${ }^{86}$

The buckling actuated transfer printing technique ${ }^{82}$ utilizes a stamp with a surface angled cylinder pillar to modulate the adhesion strength. During the retrieval process, a gentle force is applied on the stamp to ensure the conformal contact between the stamp and the ink followed by retracting the stamp rapidly to retrieve the ink from the donor substrate. During the printing process, a vertical displacement is applied until the pillar is compressed to buckle, which causes the delamination of the pillar and leaves only the tip to contact with the ink, thus reducing the stamp/ink adhesion and facilitating the release of the ink. It is found that the adhesion switchability can reach up to 39 for the buckling actuated transfer printing technique.

The above gecko-inspired transfer printing techniques focus on the reduction of the adhesion at the stamp/ink interface for printing process and give less attention on the increase of adhesion for the retrieval process. Although the intrinsic adhesion can usually satisfy the retrieval requirements for most materials, the improvement of adhesion is needed to further expand the scope of applications. Inspired by the gecko's toe pad with strong adhesion strength enabled by spatulate tips, ${ }^{87} \mathrm{Kim}$ et al. ${ }^{83}$ utilized a pedestal stamp with a rectangular post on an end pad to improve the adhesion and enhance the reliability of retrieval as shown in Fig. $6 \mathrm{~d}$. The adding of the end pad changes the delamination diagram, ${ }^{88}$ and initiates the crack at the center rather than on the edge of the pad. Compared with a flat punch, the pedestal stamp design offers enhanced adhesion $(15 \times$, $1600 \mathrm{kPa}$, see Table 1), which expands the breadth of retrievable materials.

The microstructures of the stamp in the above mentioned gecko-inspired transfer printing techniques are quite simple compared to the hierarchical structures of the natural setal arrays of geckos and none of them can offer the same combination of the remarkable properties of the gecko adhesive. Jeong et al. ${ }^{84}$ fabricated stamp covered by the seta array harvested from the

(a)
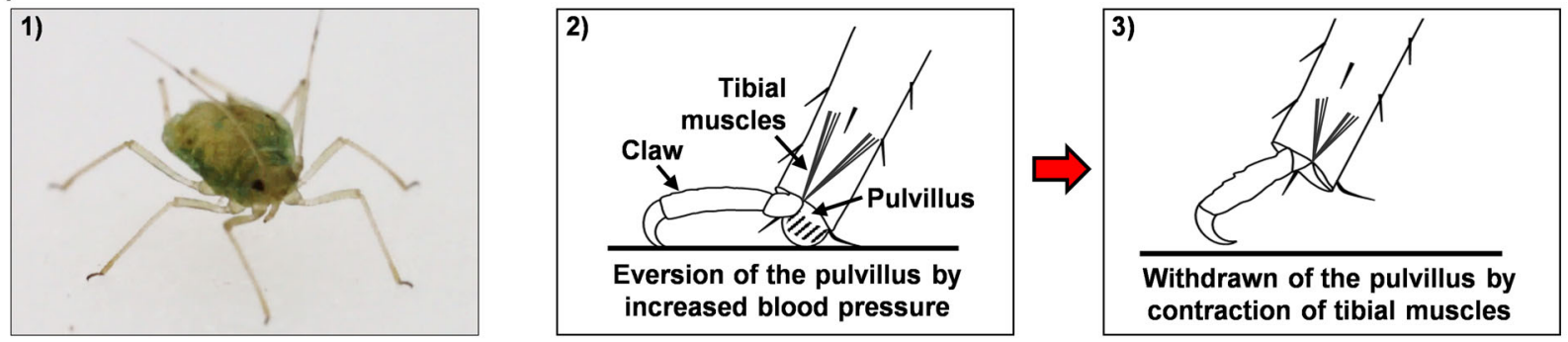

(b)
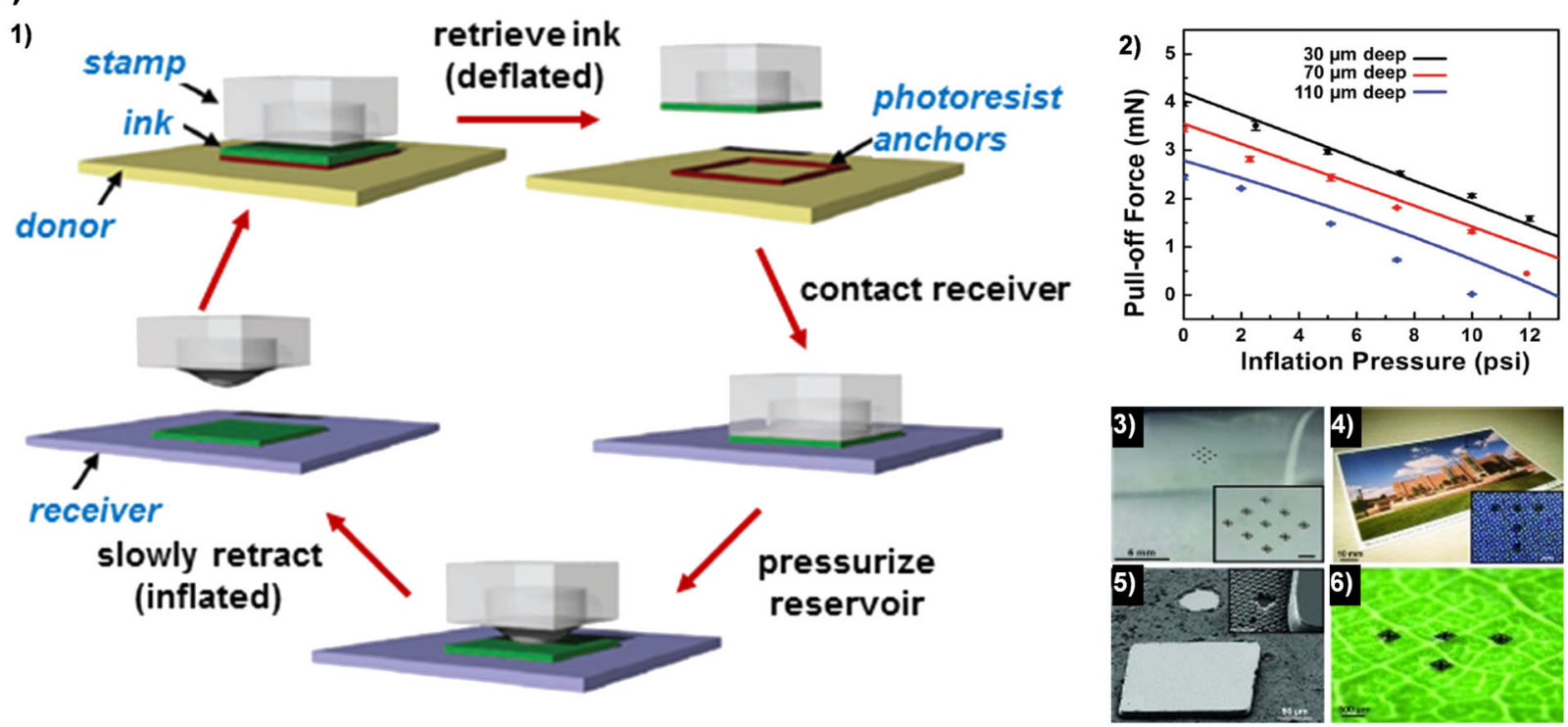

Fig. 7 Aphid-inspired transfer printing techniques: inflatable stamp. a Image of an aphid and structure of the aphid adhesion system. (1) Optical image of an aphid clinging to a flat surface; (2) Aphid adhesion organ (pulvillus) is everted to drive the mechanical sagging of the pulvillus and enlarge the contacting area, resulting in strong adhesion. (3) Aphid adhesion organ (pulvillus) is withdrawn by contraction of tibial muscles, which drives the retraction of the pulvillus and diminishes the contacting area, leading to weak adhesion. $\mathbf{b}$ Transfer printing technique using an inflatable stamp: (1) Transfer printing process; (2) Experimentally and theoretically predicted decrease in stamp/silicon interfacial adhesion (maximum pull-off force) with the inflation pressure and (3-6) capacity demonstrations of transfer printing. Adapted with permission from ref. ${ }^{91}$ 
natural gecko's toe. The retraction of the gecko seta array stamp along the proximal direction enables the retrieval of the inks. In contrast, the retraction along the distal direction requires nearly no adhesion force for delamination, thus facilitating the printing of inks onto various challenging substrates such as the leaves of japonicum, the back and the wing of an insect.

Gecko-inspired transfer printing techniques are very effective in both the maximum adhesion strength and adhesion switchability. Besides, these transfer printing techniques can minimize the influence of the transfer printing process on the performance of the devices, as shown in Figs. $6 e^{83}, f^{84}$ where the I-V characteristics of the LED transfer-printed by a pedestal stamp ${ }^{83}$ and solar cell transfer-printed by a gecko seta stamp ${ }^{84}$ show nearly no performance difference with those before transfer printing. However, the fabrication of these fibrillar structures usually requires micro/nanofabrication equipment which is not commonly available and the fabrication process is quite complicated and costly. ${ }^{79}$ In addition, the shear force introduced by the lateral movement or buckling may cause the lateral sliding of the ink, leading to misalignments.

\section{Aphid-inspired transfer printing technique}

The other adhesion control strategy that has been widely used in animal kingdom is called the pneumatic mechanism, where the adhesion is modulated by changing the effective stiffness and topography of the smooth adhesive pads. The aphid is a typical animal utilizing this adhesion control strategy, ${ }^{89,90}$ as illustrated in Fig. 7a. When adhering to or releasing from a smooth surface (Fig. 7a-1), the aphid pulvillus enlarges or diminishes the contacting areas in a reversible fashion that induces corresponding changes in adhesion strength. ${ }^{71}$ Inspired by the aphid's tunable and reversible adhesive pad, many aphid-inspired transfer printing techniques based on contacting area change have been developed such as transfer printing technique using an inflatable stamp, ${ }^{91}$ surface-relief assisted transfer printing technique, ${ }^{71}$ etc.

Carlson et al. ${ }^{91}$ developed a transfer printing technique using an inflatable stamp, which is featured by micro-channels in the stamp, open reservoirs on the bottom surface, and a thin adhesive membrane encapsulating the reservoirs. The thin adhesive membrane can be pressured in a controlled manner via inflation to induce various levels of surface deformation. The deformed thin membrane reduces its contact area with the ink, and thus the adhesion at stamp/ink interface. Figure $7 \mathrm{~b}-1$ illustrates the transfer printing process using the inflatable stamp. Large contact area and high adhesion between the flat adhesive membrane and the ink enable the retrieval of the ink. During the printing process, the adhesive membrane is pressurized, leading to a small contact area and weak adhesion for printing. With special designs, the stamp reservoirs (or the adhesive membrane) can be individually addressed, which facilitates selective/programmable printing. The adhesion strength at the stamp/ink interface decreases with the increased pressure (Fig. 7b-2), which offers continuously tunable adhesion with high accuracy. The capability of this transfer printing technique has been demonstrated by printing silicon plates onto various challenging substrates, such as plastic sheets (Fig. 7b-3), paper-based material (Fig. 7b-4), textured surfaces (Fig. 7b-5), and organic matter (Fig. 7b-6). The inflatable stamp has provided valuable proof for the selective/programmable printing, but the complexity of the fabrication process and the small packing density due to the connection requirements between the air pipe and each actuation site via the microchannels limit its practical applications in large scale twodimensional arrays. A simple yet robust design ${ }^{90}$ is introduced by adding the magnetic particles into the reservoirs to drive the membrane deformations and modulate the adhesion via the external magnetic field. The built-in magnetic driving system eliminates the need for the connection to air pipes and responses very quickly.

Kim et al. ${ }^{71}$ developed a surface-relief assisted transfer printing technique that utilizes a stamp with a simpler design but a greater contact area change ( 80 vs. $0.007 \%$ ) induced by the collapse and elastic recovery of micro-pyramid structures on the stamp surface. The transfer printing process is illustrated in Fig. 8a-1. Before the retrieval, a large preload is applied on the stamp to collapse the micro-pyramid structures (Fig. 8a-2) and yields a large contact area between the stamp and the ink. To ensure a successful retraction, the stamp must be retracted quickly followed by placing it on top of the donor substrate to avoid the delamination of the ink from the stamp before the printing starts. During the printing process, the elastic restoring force brings the collapsed micro-pyramid structures back to its original geometry, leaving only the tip contact with the interface (Fig. 8a-3). The slow removal of the stamp leaves the ink on the receiver substrate and completes the transfer printing process. This stamp with surface relief can provide extremely high adhesion switchability of more than three orders as shown in Fig. 8a-4. This capability facilitates robust transfer printing of active materials onto nearly any surfaces. Several parameters are crucial for successful implementation including the work of adhesion, the modulus of the stamp, and most importantly, the height of the micro-pyramid relief. A tall micro-pyramid structure may fail to retrieve while a short micropyramid structure may fail to print due to the large and small restoring forces, respectively. The maximum and minimum height of micro-pyramid structures have been obtained from threedimensional mechanics analysis, ${ }^{71,92}$ which are useful to guide the design of surface-relief assisted transfer printing technique.

The elastic recovery of the collapsed micro-pyramid structures is passive and time sensitive due to the viscoelastic properties of PDMS, which may yield unexpected tilt, rotation and even drop of inks during the transfer printing process. Instead of using PDMS stamp, shape memory polymer (SMP) stamp is introduced to improve the controllability and the reliability ${ }^{93,94}$ of transfer printing as shown in Fig. 8b. SMPs have the characteristics of significant elastic modulus change across the polymer's glass transition temperature $(\mathrm{Tg})$, temporary shape locking and permanent shape recovery upon heating. The SMP stamp is of the same design that features micro-pyramid structures on the surface, which defines the permanent shape of the stamp. At the beginning, the SMP stamp is heated above its $\mathrm{Tg}$ and becomes compliant, a preload is applied to drive the micro-pyramid structures to collapse onto the ink. The collapsed SMP stamp is then cooled down to lock the temporary shape followed by the reliable retrieval of inks from the donor substrate. Upon printing, the SMP stamp is heated above its Tg to recover its permanent shape, which leaves only tip contact and ensures a highly reliable printing. The introducing of laser pulse to heat the local region enables selective/programmable printing as shown in Fig. $8 c^{51,52}$ The only problem with the SMP stamp lies in the high adhesion upon heating, which is unfavorable for the printing. Figure $8 \mathrm{~d}$ shows some devices and structures fabricated by the surfacerelief-assisted transfer printing technique, such as carbon nanotube field effect transistor (CNFET) with $100 \mathrm{~nm}$ thick air gap dielectric fabricated using a heavily doped silicon platelet, $^{71}$ bendable and twistable imbricate architecture design, ${ }^{95}$ seriesconnected array of pressure activated connections on flexed $\mathrm{PEN}^{96}$ and "MECHSE" pattern of gold-coated $\mathrm{Si}$ inks on a curved PDMS substrate using multiple selective printing steps. ${ }^{51}$

\section{Perspectives and challenges}

Transfer printing techniques facilitate the heterogeneous integration of dissimilar micro-materials and nano-materials into spatially organized, functional arrangements in two-dimensional and threedimensional layouts. It decouples the device fabrication and 
integration process and brings many novel application opportunities for flexible and stretchable inorganic electronics, which are impossible by conventional techniques (e.g., etching and deposition). In the past decade, transfer printing techniques have received and continuously attracted attention from academia and industry due to their wide applications in the area of flexible and stretchable inorganic electronics and other areas beyond flexible and stretchable inorganic electronics, such as reconfigurable systems, ${ }^{97}$ assembly of heterogeneous materials, ${ }^{25}$ etc.

The performances of the main transfer printing techniques are summarized and compared in Table 1. Transfer printing techniques assisted with surface chemistry or glue and transfer printing techniques using solvent releasable tapes or thermal releasable tapes provide exceptional maximum adhesion and switchability, which makes them very reliable transfer printing protocols. However, the transfer printing process is usually destructive to the stamp interface with the adhesion not reversible and stamp not reusable. Moreover, the surface residues on the devices may degrade the device performance. Advanced transfer printing

(a)

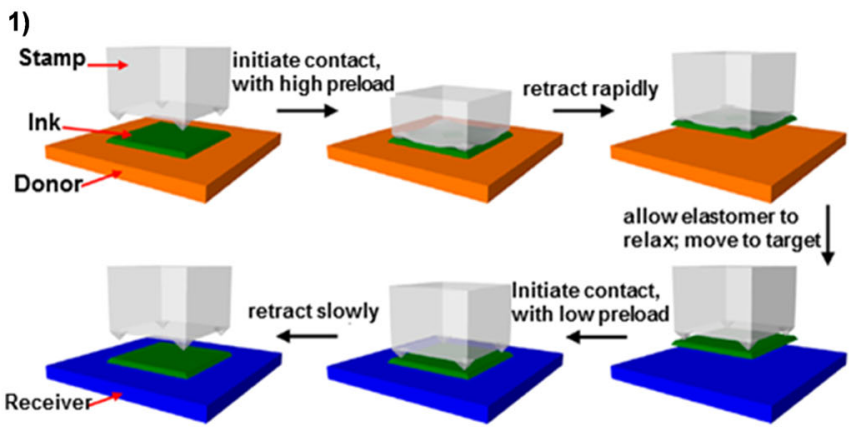

techniques based on reversible dry adhesion provide a good solution to the above limitations by offering repeatability without leaving any residues on devices. Among them, the kinetically controlled transfer printing technique provides the most versatile and convenient scheme but with limited adhesion modulation range. Laser-driven non-contact transfer printing is the only transfer printing method providing non-contact printing but the undesirable stamp surface damage due to the high temperature needs to be carefully addressed. Gecko-inspired transfer printing techniques outperform the formerly mentioned techniques but the fabrication of the stamp with surface micro-structures poses challenges on the cost. On the contrary, aphid-inspired transfer printing techniques are of simpler design and can provide great adhesion switchability. Selective/non-selective transfer printing processes with local, non-contact outer stimuli (e.g., laser beam) could enhance the versatility and applicability of transfer printing techniques.

Surface chemistry and glue assisted transfer printing techniques and kinetically controlled transfer printing technique are widely

\section{(b)}

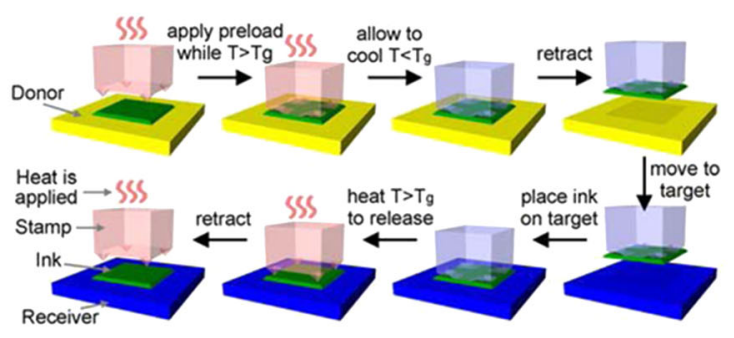

(c)

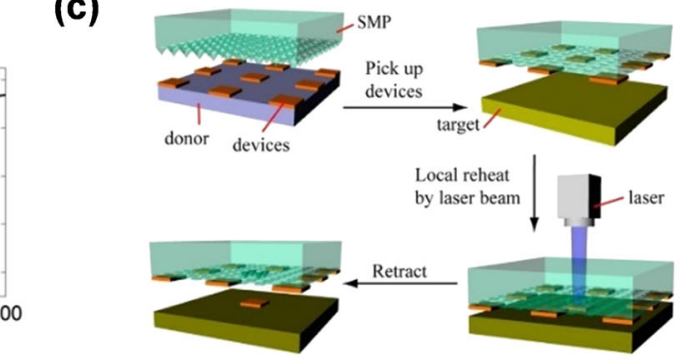

(d)
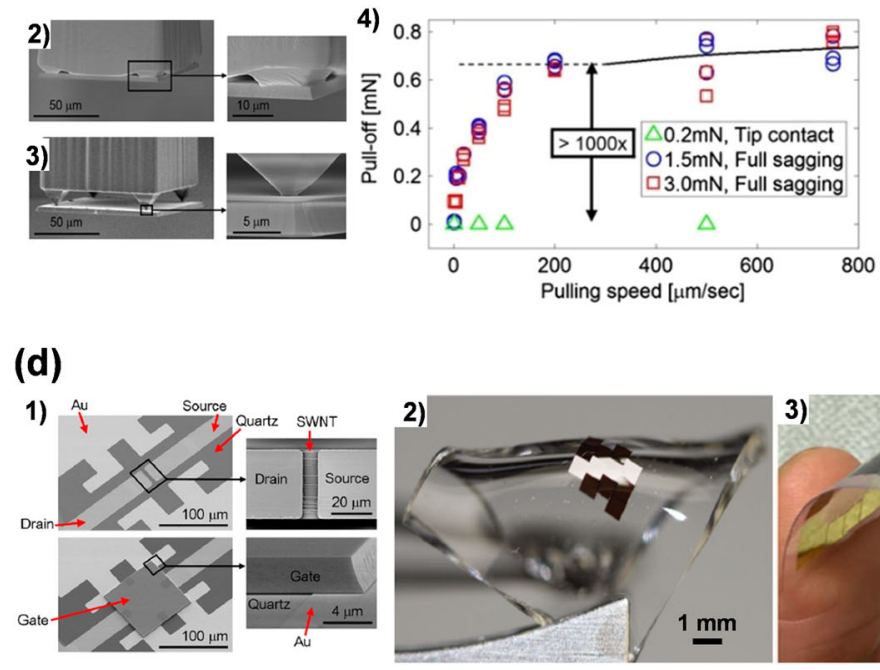

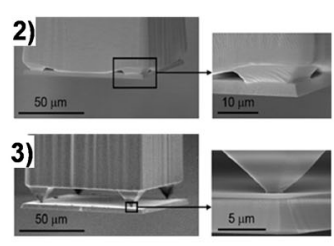

Pulling speed $[\mu \mathrm{m} / \mathrm{sec}]$

Fig. 8 Aphid-inspired transfer printing techniques: surface-relief assisted transfer printing. a Surface-relief assisted transfer printing technique. (1) Implementation of the aphid-inspired, surface-relief assisted transfer printing for deterministic assembly; SEM images of the (2) collapsed stamp and (3) recovered stamp with silicon platelets $(3 \mu \mathrm{m}$ thick; $100 \times 100 \mu \mathrm{m})$ on their surfaces; (4) Force required to remove a microtip surface from the silicon, as a function of retraction speed for three different preload cases, simulating the steps of retrieval (1.5, $3 \mathrm{mN})$ and printing $(0.2 \mathrm{mN})$ process. Adapted with permission from ref. ${ }^{71} \mathbf{b}$ Schematic illustration of the bonding/debonding between the SMP stamp surface and a substrate, where the recovery of the micro-pyramid structures is controlled by global heating. Reprinted with permissions from ref. ${ }^{93} \mathrm{c}$ Schematic illustration of the programmable printing process via automated laser writing on a micropatterned shape memory polymer stamp. Reprinted with permissions from ref. ${ }^{52} \mathbf{d}$ Devices and structures fabricated by surface-relief assisted transfer printing. (1) Carbon nanotube field effect transistor (CNFET) with $100 \mathrm{~nm}$ thick air gap dielectric fabricated using surface-relief assisted transfer printing a heavily doped silicon platelet. Reprinted with permissions from ref. ${ }^{71}$ (2) Bendable and twistable imbricate architecture design sample realized by surface-relief assisted transfer printing. Reprinted with permissions from ref. ${ }^{95}$ (3) Series-connected array of pressure activated connections on flexed PEN fabricated using surface-assisted transfer printing. Reprinted with permissions from ref. ${ }^{96}$ (4) "MECHSE" pattern of gold-coated Si inks on a curved PDMS substrate using multiple selective printing steps with laser-driven, surface-relief assisted SMP stamp. Reprinted with permissions from ref. ${ }^{51}$ 
exploited and applied in the fabrication of flexible and stretchable electronics. However, as newly developed advanced transfer printing techniques, the applications of laser-driven non-contact transfer printing technique and gecko-inspired or aphid-inspired transfer printing techniques in the fabrication of electronics (i.e., sensors, displays and circuits) are rarely reported. Existing reports mainly focus on the demonstration of the viability of these novel ideas, the investigation of related mechanism and optimization. Future work should provide more proofs on the feasibility of these transfer printing techniques in the fabrication of electronic devices.

Although there are significant advances of transfer printing techniques in terms of the ink material compatibility, the receiver substrate's material and geometry tolerance, the adhesion switchability and the reliability, several challenges still exist for future transfer printing techniques:

(1) Scalability towards nano-scale. The dimension of inks that existing transfer printing techniques can enable is on the order of micro-scale. It is hard to achieve a high yield of transfer printing as the ink becomes thinner than $100 \mathrm{~nm}$ and smaller than $1 \mu \mathrm{m}$. Future transfer printing techniques should be able to extend the ink size to nano-scale in a controllable manner.

(2) High parallelism and large scale. A high throughput for large scale manufacturing is desired for transfer printing techniques in industry. The increase of stamp area and the parallelism at each printing cycle may provide a solution, but the avoiding of missed retrievals and prints becomes even harder as the parallelism increases. Attempts have been made including the incorporation of angled post into the roll-to-roll process ${ }^{86}$ and the combination of advanced transfer printing techniques with an automated platform $^{31,98,99}$ in selective/programmable mode.

(3) Direct three-dimensional transfer printing capability. Current transfer printing techniques are incompatible with receiver substrates with complex geometries. Although the transfer printing of individual device onto any surface ${ }^{72,73}$ has been realized by the laser-driven non-contact transfer printing technique, it is hard to print the ink array on any surface with high throughput. For the applications in bio-integrated devices that require intimate contact with tissues of complex topologies, ${ }^{100}$ the directly three-dimensional transfer printing techniques with high throughput is desired.

\section{CONCLUSION}

This paper presents a brief review on the advances of transfer printing techniques for flexible and stretchable inorganic electronics. The general trend of transfer printing technique is toward to the harmless, reusable, more versatile and controllable protocols. Transfer printing techniques ranging from surface chemistry and glue assisted transfer printing technique, where the interfacial adhesion is modulated non-reversibly by surface chemistry, to advanced transfer printing techniques (including kinetically controlled transfer printing technique, laser-driven non-contact transfer printing technique, gecko-inspired transfer printing technique, and aphid-inspired transfer printing technique), where the interfacial adhesion is tunable and reversible by external physical stimuli, are overviewed. The performances of these transfer printing techniques are summarized and compared. Some perspectives and challenges are presented for future developments of transfer printing techniques.

\section{DATA AVAILABILITY}

All data are available within the article or available from the authors upon reasonable request.

\section{ACKNOWLEDGEMENTS}

The authors acknowledge the supports from the Zhejiang Provincial Natural Science Foundation of China (Grant No. LR15A020001), the National Basic Research Program (Grant No. 2015CB351901), the National Natural Science Foundation of China (Grant Nos. 11372272, 11622221, and 11621062), the Shenzhen Science and Technology Program (Grant No. JCY20170816172454095), and the Fundamental Research Funds for the Central Universities.

\section{AUTHOR CONTRIBUTIONS}

C. L., S. Z., and C. W. studied the literatures. C. L. and J. S. wrote the paper.

\section{ADDITIONAL INFORMATION}

Competing interests: The authors declare no competing interests.

Publisher's note: Springer Nature remains neutral with regard to jurisdictional claims in published maps and institutional affiliations.

\section{REFERENCES}

1. Sun, Y., Choi, W. M., Jiang, H., Huang, Y. Y. \& Rogers, J. A. Controlled buckling of semiconductor nanoribbons for stretchable electronics. Nat. Nanotechnol. 1, 201-207 (2006).

2. Kim, D. H. et al. Materials and noncoplanar mesh designs for integrated circuits with linear elastic responses to extreme mechanical deformations. Proc. Natl Acad. Sci. 105, 18675-18680 (2008).

3. Song, Z. et al. Origami lithium-ion batteries. Nat. Commun. 5, 3140 (2014).

4. Cho, Y. et al. Engineering the shape and structure of materials by fractal cut. Proc. Natl Acad. Sci. 111, 17390-17395 (2014).

5. Yu, K. J., Yan, Z., Han, M. \& Rogers, J. A. Inorganic semiconducting materials for flexible and stretchable electronics. npj Flex. Electron. 1, 4 (2017).

6. Ko, H. C. et al. A hemispherical electronic eye camera based on compressible silicon optoelectronics. Nature 454, 748-753 (2008).

7. Song, Y. M. et al. Digital cameras with designs inspired by the arthropod eye. Nature 497, 95-99 (2013).

8. Ko, H. C. et al. Curvilinear electronics formed using silicon membrane circuits and elastomeric transfer elements. Small 5, 2703-2709 (2009).

9. Kim, D. H. et al. Materials for multifunctional balloon catheters with capabilities in cardiac electrophysiological mapping and ablation therapy. Nat. Mater. 10, 316-323 (2011).

10. Yan, Z. et al. Thermal release transfer printing for stretchable conformal bioelectronics. Adv. Sci. 4, 1700251 (2017).

11. Hyun-Joong, $\mathrm{C}$. et al. Stretchable, multiplexed $\mathrm{pH}$ sensors with demonstrations on rabbit and human hearts undergoing ischemia. Adv. Healthc. Mater. 3, 59-68 (2014).

12. Jae-Woong, J. et al. Materials and optimized designs for human-machine interfaces via epidermal electronics. Adv. Mater. 25, 6839-6846 (2013).

13. Yeo, W. H. et al. Multifunctional epidermal electronics printed directly onto the skin. Adv. Mater. 25, 2773-2778 (2013).

14. Kim, D.-H. et al. Epidermal electronics. Science 333, 838-843 (2011).

15. Hwang, S. W. et al. 25th anniversary article: materials for high-performance biodegradable semiconductor devices. Adv. Mater. 26, 1992-2000 (2014).

16. Jung, Y. H. et al. High-performance green flexible electronics based on biodegradable cellulose nanofibril paper. Nat. Commun. 6, 7170 (2015).

17. Hwang, S. W. et al. A physically transient form of silicon electronics. Science 337, 1640-1644 (2012).

18. Kim, B. H. et al. Dry transient electronic systems by use of materials that sublime. Adv. Funct. Mater. 27, 1606008 (2017).

19. Kim, H. S. et al. Unusual strategies for using indium gallium nitride grown on silicon (111) for solid-state lighting. Proc. Natl Acad. Sci. 108, 10072-11077 (2011).

20. Park, S. I. et al. Printed assemblies of inorganic light-emitting diodes for deformable and semitransparent displays. Science 325, 977-981 (2009).

21. Kim, T. H. et al. Full-colour quantum dot displays fabricated by transfer printing. Nat. Photonics 5, 176-182 (2011).

22. Kim, B. H. et al. Multilayer transfer printing for pixelated, multicolor Quantum dot light-emitting diodes. Acs Nano 10, 4920-4925 (2016).

23. Yoon, J. et al. Ultrathin silicon solar microcells for semitransparent, mechanically flexible and microconcentrator module designs. Nat. Mater. 7, 907-915 (2008).

24. Baca, A. J. et al. Compact monocrystalline silicon solar modules with high voltage outputs and mechanically flexible designs. Energy Environ. Sci. 3, 208-211 (2010). 
25. Lee, S. et al. Heterogeneously assembled metamaterials and metadevices via 3D modular transfer printing. Sci. Rep. 6, 27621 (2016).

26. $\mathrm{Yu}, \mathrm{C}$. et al. Electronically programmable, reversible shape change in two and three dimensional hydrogel structures. Adv. Mater. 25, 1541-1546 (2013).

27. Tao, H. et al. Silk-based conformal, adhesive, edible food sensors. Adv. Mater. 24, 1067-1072 (2012).

28. Kim, D.-H. et al. Strechable and foldable silicon integrated circuits. Science $\mathbf{3 2 0}$, 507-511 (2008)

29. Kim, D.-H. et al. Dissolvable films of silk fibroin for ultrathin, conformal biointegrated electronics. Nat. Mater. 9, 511-517 (2010).

30. Kim, R.-H. et al. Waterproof AllnGaP optoelectronics on stretchable substrates with applications in biomedicine and robotics. Nat. Mater. 9, 929-937 (2010).

31. Carlson, A., Bowen, A. M., Huang, Y., Nuzzo, R. G. \& Rogers, J. A. Transfer printing techniques for materials assembly and micro/nanodevice fabrication. Adv. Mater. 24, 5284-5318 (2012).

32. Menard, E., Lee, K. J., Khang, D.-Y., Nuzzo, R. G. \& Rogers, J. A. A printable form of silicon for high performance thin film transistors on plastic substrates. Appl. Phys. Lett. 84, 5398-5400 (2004).

33. Loo, Y., Willett, R. L., Baldwin, K. W. \& Rogers, J. A. Interfacial chemistries for nanoscale transfer printing. J. Am. Chem. Soc. 124, 7654 (2002).

34. Smythe, E. J., Dickey, M. D., Whitesides, G. M. \& Capasso, F. A technique to transfer metallic nanoscale patterns to small and non-planar surfaces. Acs Nano 3, 59-65 (2009).

35. Dae-Hyeong, K. et al. Flexible electronics: ultrathin silicon circuits with strainisolation layers and mesh layouts for high-performance electronics on fabric, vinyl, leather, and paper. Adv. Mater. 21, 703-3707 (2009).

36. Choi, C. et al. Human eye-inspired soft optoelectronic device using high-density $\mathrm{MoS}_{2}$-graphene curved image sensor array. Nat. Commun. 8, 1664 (2017).

37. Jun-ho, C., Kyung-Ho, K., Se-Jin, C. \& Hong, H. L. Whole device printing for full colour displays with organic light emitting diodes. Nanotechnology 17, 2246 (2006).

38. Blanchet, G. B., Fincher, C. R. \& Gao, F. Polyaniline nanotube composites: a highresolution printable conductor. Appl. Phys. Lett. 82, 1290-1292 (2003).

39. Kim, S. J. et al. Multifunctional cell-culture platform for aligned cell sheet monitoring, transfer printing, and therapy. ACS Nano 9, 2677-2688 (2015).

40. Joo, K. S. et al. Stretchable and transparent biointerface using cell-sheetgraphene hybrid for electrophysiology and therapy of skeletal muscle. Adv. Funct. Mater. 26, 3207-3217 (2016).

41. Ahn, J. $\mathrm{H}$. et al. Transfer Printing Techniques and Inorganic Single-Crystalline Materials for Flexible and Stretchable Electronics. (Wiley, Hoboken, 2008).

42. Yoon, J. et al. Heterogeneously integrated optoelectronic devices enabled by micro-transfer printing. Adv. Opt. Mater. 3, 1313-1335 (2015).

43. Ravikumar, V., Yi, N., Vepachedu, V. \& Cheng, H. in Industrial Internet of Things: Cybermanufacturing Systems (eds S. Jeschke, C. Brecher, H. Song, \& D. B. Rawat) 671-690 (Springer, New York, 2017).

44. Kimlee, H. J., Carlson, A., Grierson, D. S., Rogers, J. A. \& Turner, K. T. Interface mechanics of adhesiveless microtransfer printing processes. J. Appl. Phys. 115, 2332-2271 (2014)

45. Sun, Y. et al. Photolithographic route to the fabrication of micro/nanowires of III-V semiconductors. Adv. Funct. Mater. 15, 30-40 (2005).

46. Wong, W. S. et al. Fabrication of thin-film InGaN light-emitting diode membranes by laser lift-off. Appl. Phys. Lett. 75, 1360-1362 (1999).

47. Wong, W. S. et al. InxGa1-xN light emitting diodes on Si substrates fabricated by Pd-In metal bonding and laser lift-off. Appl. Phys. Lett. 77, 2822-2824 (2000)

48. Meitl, M. A. et al. Transfer printing by kinetic control of adhesion to an elastomeric stamp. Nat. Mater. 5, 33-38 (2006).

49. Cho, S., Kim, N., Song, K. \& Lee, J. Adhesiveless transfer printing of ultrathin microscale semiconductor materials by controlling the bending radius of an elastomeric stamp. Langmuir 32, 7951-7957 (2016).

50. Ahmed, N., Dagdeviren, C., Rogers, J. A. \& Ferreira, P. M. Active polymeric composite membranes for localized actuation and sensing in microtransfer printing. J. Micro Syst. 24, 1016-1028 (2015).

51. Eisenhaure, J. \& Kim, S. Laser-driven shape memory effect for transfer printing combining parallelism with individual object control. Adv. Mater. Technol. 1, 1600098 (2016)

52. Huang, Y. et al. Direct laser writing-based programmable transfer printing via bioinspired shape memory reversible adhesive. Acs Appl. Mater. Interfaces 8 , 35628-35633 (2016)

53. Feng, X. et al. Competing fracture in kinetically controlled transfer printing. Langmuir 23, 12555-12560 (2007).

54. Kim, T.-H. et al. Kinetically controlled, adhesiveless transfer printing using microstructured stamps. Appl. Phys. Lett. 94, 113502 (2009).

55. Carlson, A. et al. Shear-enhanced adhesiveless transfer printing for use in deterministic materials assembly. Appl. Phys. Lett. 98, 264104 (2011).
56. Sun, Y. \& Rogers, J. A. Fabricating semiconductor nano/microwires and transfer printing ordered arrays of them onto plastic substrates. Nano Lett. 4, 1953-1959 (2004).

57. Duffy, D. C., McDonald, J. C., Schueller, O. J. A. \& Whitesides, G. M. Rapid prototyping of microfluidic systems in poly(dimethylsiloxane). Anal. Chem. 70, 4974-4984 (1998).

58. Lee, K. J. et al. Large-area, selective transfer of microstructured silicon: a printing-based approach to high-performance thin-film transistors supported on flexible substrates. Adv. Mater. 17, 2332-2336 (2005).

59. Park, J. S. et al. Microtransfer printing of metal ink patterns onto plastic substrates utilizing an adhesion-controlled polymeric donor layer. J. Micromech. Microeng. 26, 065008 (2016).

60. Menard, E., Nuzzo, R. G. \& Rogers, J. A. Bendable single crystal silicon thin film transistors formed by printing on plastic substrates. Appl. Phys. Lett. 86, 093507, https://doi.org/10.1063/1.1866637 (2005).

61. Baca, A. J. et al. Printable single-crystal silicon micro/nanoscale ribbons, platelets and bars generated from bulk wafers. Adv. Funct. Mater. 17, 3051-3062 (2007).

62. Kim, T. et al. Thin film receiver materials for deterministic assembly by transfer printing. Chem. Mater. 26, 3502-3507 (2014).

63. Loo, Y.-L. et al. High-resolution transfer printing on GaAs surfaces using alkane dithiol monolayers. J. Vac. Sci. Technol. B 20, 2853-2856 (2002).

64. Choi, M. K. et al. Wearable red-green-blue quantum dot light-emitting diode array using high-resolution intaglio transfer printing. Nat. Commun. 6, 7149 (2015).

65. Jeong, J. W. et al. High-resolution nanotransfer printing applicable to diverse surfaces via interface-targeted adhesion switching. Nat. Commun. 5, 5387 (2014).

66. Sim, K. et al. High fidelity tape transfer printing based on chemically induced adhesive strength modulation. Sci. Rep. 5, 16133 (2015).

67. Lee, C. H., Kim, D. R. \& Zheng, X. Fabricating nanowire devices on diverse substrates by simple transfer-printing methods. Proc. Natl Acad. Sci. 107 9950-9955 (2010)

68. Ahn, J.-H. et al. Heterogeneous three-dimensional electronics by use of printed semiconductor nanomaterials. Science 314, 1754-1757 (2006).

69. Chen, H., Feng, X. \& Chen, Y. Directionally controlled transfer printing using micropatterned stamps. Appl. Phys. Lett. 103, 151607 (2013).

70. Yoon, J. et al. GaAs photovoltaics and optoelectronics using releasable multilayer epitaxial assemblies. Nature 465, 329-333 (2010).

71. Kim, S. et al. Microstructured elastomeric surfaces with reversible adhesion and examples of their use in deterministic assembly by transfer printing. Proc. Nat Acad. Sci. 107, 17095-17100 (2010).

72. Saeidpourazar, R. et al. Laser-driven micro transfer placement of prefabricated microstructures. J. Micro Syst. 21, 1049-1058 (2012).

73. Saeidpourazar, R., Sangid, M. D., Rogers, J. A. \& Ferreira, P. M. A prototype printer for laser driven micro-transfer printing. J. Manuf. Process. 14, 416-424 (2012).

74. Li, R. et al. Thermo-mechanical modeling of laser-driven non-contact transfer printing: two-dimensional analysis. Soft Matter 8, 7122-7127 (2012).

75. Li, R. et al. Axisymmetric thermo-mechanical analysis of laser-driven non-contact transfer printing. Int. J. Fract. 176, 189-194 (2012).

76. Gao, Y., Li, Y., Li, R. \& Song, J. An accurate thermomechanical model for laserdriven microtransfer printing. J. Appl. Mech. 84, 064501 (2017).

77. Autumn, K., Dittmore, A., Santos, D., Spenko, M. \& Cutkosky, M. Frictional adhesion: a new angle on gecko attachment. J. Exp. Biol. 209, 3569-3579 (2006).

78. Autumn, K. Properties, Principles, and Parameters of the Gecko Adhesive System. (Springer, Cham, 2006).

79. Brodoceanu, D., Bauer, C. T., Kroner, E., Arzt, E. \& Kraus, T. Hierarchical bioinspired adhesive surfaces-a review. Bioinspir. Biomim. 11, 051001 (2016).

80. Rizzo, N. W. et al. Characterization of the structure and composition of gecko adhesive setae. J. R. Soc. Interface 3, 441-451 (2006).

81. Yoo, B., Cho, S., Seo, S. \& Lee, J. Elastomeric angled microflaps with reversible adhesion for transfer-printing semiconductor membranes onto dry surfaces. ACS Appl. Mater. Interfaces 6, 19247-19253 (2014).

82. Mengüç, Y., Yang, S. Y., Kim, S., Rogers, J. A. \& Sitti, M. Gecko-inspired controllable adhesive structures applied to micromanipulation. Adv. Funct. Mater. 22, 1246-1254 (2012)

83. Kim, S. et al. Enhanced adhesion with pedestal-shaped elastomeric stamps for transfer printing. Appl. Phys. Lett. 100, 171909 (2012).

84. Jeong, J., Kim, J., Song, K., Autumn, K. \& Lee, J. Geckoprinting: assembly of microelectronic devices on unconventional surfaces by transfer printing with isolated gecko setal arrays. J. R. Soc. Interface 11, 20140627 (2014).

85. Hutchinson, J. W. \& Suo, Z. in Advances in Applied Mechanics Vol. 29 (eds J. W. Hutchinson \& T. Y. Wu) 63-191 (Elsevier, 1991).

86. Yang, S. Y. et al. Elastomer surfaces with directionally dependent adhesion strength and their use in transfer printing with continuous roll-to-roll applications. Adv. Mater. 24, 2117-2122 (2012). 
87. Kim, S. \& Sitti, M. Biologically inspired polymer microfibers with spatulate tips as repeatable fibrillar adhesives. Appl. Phys. Lett. 89, 261911 (2006).

88. Carbone, G., Pierro, E. \& Gorb, S. N. Origin of the superior adhesive performance of mushroom-shaped microstructured surfaces. Soft Matter 7, 5545-5552 (2011).

89. Lees, A. D. \& Hardie, J. The organs of adhesion in the Aphid Megoura Viciae. J. Exp. Biol. 136, 209-228 (1988).

90. Linghu, C. et al. Rapidly tunable and highly reversible bio-inspired dry adhesion for transfer printing in air and vacuum. Submitted (2018).

91. Carlson, A. et al. Active, programmable elastomeric surfaces with tunable adhesion for deterministic assembly by transfer printing. Adv. Funct. Mater. 22, 4476-4484 (2012).

92. $\mathrm{Wu}$, J. et al. Mechanics of reversible adhesion. Soft Matter 7, 8657-8662 (2011).

93. Eisenhaure, J. D. et al. The use of shape memory polymers for microassembly by transfer printing. J. Micro Syst. 23, 1012-1014 (2014).

94. Xue, Y. et al. A theoretical model of reversible adhesion in shape memory surface relief structures and its application in transfer printing. J. Mech. Phys. Solids 77, 27-42 (2015).

95. Seok, K. et al. Imbricate scales as a design construct for microsystem technologies. Small 8, 901-906 (2012).

96. Prevatte, C. et al. Pressure activated interconnection of micro transfer printed components. Appl. Phys. Lett. 108, 203503 (2016).

97. Yan, Z. et al. Three-dimensional mesostructures as high-temperature growth templates, electronic cellular scaffolds, and self-propelled microrobots. Proc. Natl Acad. Sci. 114, E9455-E9464 (2017).
98. Ahmed, N., Rogers, J. A. \& Ferreira, P. M. Microfabricated instrumented composite stamps for transfer printing. J. Micro Nano-Manuf. 3, 021007 (2015).

99. Ahmed, N., Carlson, A., Rogers, J. A. \& Ferreira, P. M. Automated micro-transfer printing with cantilevered stamps. J. Manuf. Process. 14, 90-97 (2012).

100. Jang, K. I. et al. Soft network composite materials with deterministic and bioinspired designs. Nat. Commun. 6, 6566 (2015).

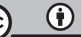

Open Access This article is licensed under a Creative Commons Attribution 4.0 International License, which permits use, sharing, adaptation, distribution and reproduction in any medium or format, as long as you give appropriate credit to the original author(s) and the source, provide a link to the Creative Commons license, and indicate if changes were made. The images or other third party material in this article are included in the article's Creative Commons license, unless indicated otherwise in a credit line to the material. If material is not included in the article's Creative Commons license and your intended use is not permitted by statutory regulation or exceeds the permitted use, you will need to obtain permission directly from the copyright holder. To view a copy of this license, visit http://creativecommons. org/licenses/by/4.0/

c C) The Author(s) 2018 